\title{
Tinnitus Correlates with Downregulation of Cortical Glutamate Decarboxylase 65 Expression But Not Auditory Cortical Map Reorganization
}

\author{
Asako Miyakawa, ${ }^{1}$ Weihua Wang, ${ }^{2}$ Sung-Jin Cho, ${ }^{3}$ Delia Li, ${ }^{1}$ Sungchil Yang, ${ }^{4}$ and Shaowen Bao ${ }^{1,2}$ \\ ${ }^{1}$ Helen Wills Neuroscience Institute, University of California, Berkeley, California 94720, ${ }^{2}$ Department of Physiology, University of Arizona, Tucson, \\ Arizona 85724, ${ }^{3}$ Department of Biology, College of Natural Sciences, Chungbuk National University, Cheongju, Chungbuk 361-763, Republic of Korea, \\ and ${ }^{4}$ Department of Biomedical Science, City University of Hong Kong, Kowloon, Hong Kong.
}

Hearing loss is the biggest risk factor for tinnitus, and hearing-loss-related pathological changes in the auditory pathway have been hypothesized as the mechanism underlying tinnitus. However, due to the comorbidity of tinnitus and hearing loss, it has been difficult to differentiate between neural correlates of tinnitus and consequences of hearing loss. In this study, we dissociated tinnitus and hearing loss in FVB mice, which exhibit robust resistance to tinnitus following monaural noise-induced hearing loss. Furthermore, knock-down of glutamate decarboxylase 65 (GAD65) expression in auditory cortex (AI) by RNA interference gave rise to tinnitus in normal-hearing FVB mice. We found that tinnitus was significantly correlated with downregulation of GAD65 in the AI. By contrast, cortical map distortions, which have been hypothesized as a mechanism underlying tinnitus, were correlated with hearing loss but not tinnitus. Our findings suggest new strategies for the rehabilitation of tinnitus and other phantom sensation, such as phantom pain.

Key words: hearing loss; inhibition; noise trauma; sensory map plasticity; tinnitus

Significance Statement

Hearing loss is the biggest risk factor for tinnitus in humans. Most animal models of tinnitus also exhibit comorbid hearing loss, making it difficult to dissociate the mechanisms underlying tinnitus from mere consequences of hearing loss. Here we show that, although both C57BL/6 and FVB mice exhibited similar noise-induced hearing threshold increase, only C57BL/6, but not FVB, mice developed tinnitus following noise exposure. Although both strains showed frequency map reorganization following noiseinduced hearing loss, only C57BL/6 mice had reduced glutamate decarboxylase 65 (GAD65) expression in the auditory cortex (AI). Knocking down GAD65 expression in the AI resulted in tinnitus in normal-hearing FVB mice. Our results suggest that reduced inhibitory neuronal function, but not sensory map reorganization, underlies noise-induced tinnitus.

\section{Introduction}

Tinnitus is the perception of phantom sounds in the absence of corresponding external sound. Its clinical etiology is diverse, including hearing loss, ototoxic drugs, ear infection, vascular ab-

Received May 14, 2019; revised Oct. 23, 2019; accepted Nov. 4, 2019.

Author contributions: A.M., S.Y., and S.B. designed research; A.M., W.W., S.-J.C., D.L., and S.Y. performed research; A.M., W.W., S.-J.C., D.L., and S.Y. analyzed data; A.M. wrote the first draft of the paper; A.M., W.W., S.-J.C., and D.L. edited the paper; A.M., S.Y., and S.B. wrote the paper.

This work was supported by the American Tinnitus Association and by the National Institute on Deafness and Other Communicative Disorders (DC009259). We thank Li. S. Zhang, Michelle Lee, Francesca Arana, and Seunghyuk D. Yang for assistance with data acquisition and Heesoo Kim, Robert Gibboni, Liberty Hamilton, Hania Köver, Jascha Sohl-Dickstein, and Alexander Zinsmaier for comments on the manuscript.

The authors declare no competing financial interests.

Correspondence should be addressed to Shaowen Bao at sbao@email.arizona.edu or Sungchil Yang at Sungchil.Yang@cityu.edu.hk.

https://doi.org/10.1523/JNEUROSCI.1117-19.2019

Copyright $\odot 2019$ the authors normality and tumors (Henry et al., 2005; Langguth et al., 2013). Among these, the biggest risk factor is hearing loss due to aging or acoustic trauma (Salvi et al., 2000; Eggermont and Roberts, 2004; Heffner and Koay, 2005; Henry et al., 2005; Elgoyhen and Langguth, 2010; Shore et al., 2016). However, despite high comorbidity, hearing loss does not always lead to tinnitus (Lockwood et al., 2002; Møller, 2011). Indeed, the prevalence of hearing loss is much higher than that of tinnitus (Lockwood et al., 2002), and the two follow different aging trajectories (Møller, 2011). It is likely that the development of tinnitus involves complex interactions between an individual's health conditions (hearing and otherwise) and genetic predispositions (Sand et al., 2007). Recent studies using mouse models showed that noise-induced tinnitus can be observed in multiple commonly used laboratory mice strains (Longenecker and Galazyuk, 2011; Middleton et al., 2011; Llano et al., 2012). Previous studies have also revealed cross- 
strain differences in tinnitus etiology with respect to age-related hearing loss (Zheng et al., 1999; Turner et al., 2006) and vulnerability to acoustic injury (Davis et al., 1999). Systematic comparisons of tinnitus in different strains of mice may inform us about neural correlates of individual differences in tinnitus susceptibility in humans. In addition, such comparisons may provide insights into the neural mechanisms underlying tinnitus.

Loss of hearing leads to reduced sensory input and lower auditory nerve discharge rates (Liberman and Kiang, 1978), which should decrease neuronal activity levels in the central auditory system. Paradoxically, spontaneous neuronal activity is elevated along the auditory pathway in animal models of noise traumainduced tinnitus (Noreña and Eggermont, 2003; Wang et al., 2011). This increase in spontaneous activity could be caused by altered neuronal excitability and imbalanced excitation and inhibition, either from enhanced neuronal excitation, or reduced inhibition as a result of hearing loss (Kotak et al., 2005; Schaette and Kempter, 2006; Sun et al., 2008; Middleton et al., 2011; Yang et al., 2011; Llano et al., 2012; Chambers et al., 2016; Resnik and Polley, 2017). In addition, noise exposure causes distortion of the cortical frequency map (Engineer et al., 2011) and changes neuronal discharge patterns (e.g., increased synchrony and more burst firing) (Noreña and Eggermont, 2003; Seki and Eggermont, 2003; Kaltenbach, 2011; Wu et al., 2016). All these noise-induced neurophysiological changes have been considered potential mechanisms of tinnitus.

A challenge in tinnitus research is to dissociate the mechanisms underlying tinnitus from mere effects of hearing loss. For example, all behavioral tests of tinnitus in animal models rely on the animal's hearing ability (Bauer and Brozoski, 2001; Lobarinas et al., 2004; Turner et al., 2006). In addition, tinnitus severity can correlate with the degree of hearing loss (Tan et al., 2007). Even in noise-exposed animals that have apparently recovered normal auditory brainstem response (ABR) thresholds, careful analysis has revealed cochlear synaptopathy and nerve degeneration, and subsequent reduction of cochlear nerve output (Kujawa and Liberman, 2009). Therefore, measurements of tinnitus could be confounded by hearing loss. To fully distinguish the neural mechanisms of tinnitus from the neurophysiological changes caused by hearing loss, tinnitus needs to be completely dissociated from hearing loss. To that end, one approach is to contrast differences between the noise-exposed animals that develop tinnitus and those that do not develop tinnitus (Pace and Zhang, 2013; Wu et al., 2016). Another approach is to screen strains of mice that are resistant to tinnitus.

In this study, we found that noise-induced hearing loss (NIHL) resulted in behavioral evidence of tinnitus (hereafter referred to as tinnitus) in C57BL/6, but not FVB mice. Similarly, NIHL resulted in reduction of GAD65 in the cortex of C57BL/6 but not FVB mice. Nevertheless, tinnitus behavior in the FVB mice could be induced by experimental knock-down of GAD65 expression. This strain difference allows us to dissociate tinnitus from hearing loss. Taking advantage of this strain difference, we show that downregulation of cortical GAD65 expression is correlated with tinnitus, whereas cortical map reorganization is correlated with hearing loss.

\section{Materials and Methods}

\section{Procedures}

All experimental procedures were reviewed and approved by the UC Berkeley and University of Arizona Animal Care and Use Committees. Three strains of mice, FVB.129P2-Pde6b ${ }^{+} \mathrm{Tyr}^{\mathrm{c}-\mathrm{ch}} / \mathrm{Ant}$, C57BL/6J, and $\mathrm{Tg}$ (dlx6a-cre)1Mekk (The Jackson Laboratory), between P60 and P100 were used in all experiments. For NIHL, C57BL/6 $(n=20$, male, P72 \pm 5 on the day of NIHL) and FVB mice $(n=20$, male, P70 \pm 5 on the day of NIHL) were used. Due to an equipment calibration issue, gap detection data from three FVB and five C57BL/6 mice were excluded, but their GAD65 data were included in the analysis. For the GAD65 knock-down experiment, two groups of FVB mice were used (experimental: $n=14$, P81 \pm 14 on the day of injection, control: $n=11$, P $67 \pm 8$ on the day of injection). ABR recordings and electrophysiological mappings were conducted on a subgroup of animals, and the number for each experiment is reported in the result section. For conditioned active avoidance behavior experiments, total of $23 \mathrm{Tg}$ (dlx6a-cre) 1 Mekk of either sex were used, and the number for each experiment is reported in the result section.

\section{NIHL and $A B R$}

Animals were anesthetized with ketamine $(100 \mathrm{mg} / \mathrm{kg}, \mathrm{IP})$ and xylazine $(10 \mathrm{mg} / \mathrm{kg}, \mathrm{IP})$, and maintained at $36.5^{\circ} \mathrm{C}$ with a homeothermic heating pad (Harvard Apparatus). Monaural NIHL was induced by playing a continuous $8 \mathrm{kHz}$ tone at $112 \mathrm{~dB}$ SPL through a custom-made piezoelectric earphone speaker to the left ear for $2 \mathrm{~h}$. The right ear was protected with sound attenuating clay. The sound level was measured with a Bruel and Kjaer 4135 condenser microphone.

Hearing thresholds were assessed under anesthesia using ABR immediately before and after $(5,15$ and $20 \mathrm{~d})$ the noise exposure procedure. ABR signals were recorded using BioSigRP software on a TDT RX5 Sys3 recording rig. Tone pips ( $3 \mathrm{~ms}$ full-cycle sine waves at $4,8,16$ and $32 \mathrm{kHz}$ at $5 \mathrm{~dB}$ intensity steps from 0 to $70 \mathrm{~dB}$ ) were delivered to a single ear through a cannulated speaker at a rate of 19 times per second. The speaker was calibrated to have $<3 \%$ harmonic distortion and flat output in the entire frequency range (Tucker-Davis Technologies SigCal32). 500 recordings were averaged for each frequency intensity pair.

ABR signals were recorded with electrodes subcutaneously inserted at three locations: behind the ear coupled with the speaker, at the vertex of the head, and near the base of the tail. ABR waveforms at each sound level were visually inspected online by the experimenter. ABR threshold was defined as the sound level at which one or more peaks were distinguishable by eye against the background activity. The amplitude and magnitude of ABR peak I to V at $60 \mathrm{~dB}$ SPL were reanalyzed offline. For offline analysis, peaks and troughs of the ABR trace were automatically identified by a custom MATLAB script. Peak latencies were defined using the time points at which peaks I to V occurred. Peak magnitude was defined as the voltage difference between a peak and preceding trough. ABR threshold, latency and magnitude at each tested frequency were compared using ANOVA and followed by post hoc $t$ tests.

\section{Behavioral test of tinnitus with a gap detection task}

During the testing session, a mouse was caged in a plastic container with a mesh lid. The container was placed on a piezoelectric disc in a sound attenuation chamber. Sounds were played through an open field speaker (Fostex FT17H) fixed above the cage. The gap detection measures the acoustic startle response elicited by a brief white noise pulse and its suppression by a preceding silent gap embedded in the background sound. Each trial starts with a carrier pure tone (frequency pseudorandomly selected from $5,7,10,14,20,28$, or $45 \mathrm{kHz}$, all at $75 \mathrm{~dB}$ SPL), played for a duration of $10-20 \mathrm{~s}$. In uncued trials, the carrier tone was followed by a startle stimulus-a $50 \mathrm{~ms}$ white noise burst at $102 \mathrm{~dB}$ SPL. In cued trials, the startle stimulus was preceded by a $50 \mathrm{~ms}$ silence, $100 \mathrm{~ms}$ before the onset. In each testing session, the animal performed a total of 500 trials ( $50 \%$ cued and 50\% uncued). After each session, we calculated the startle response ratio, which is defined as the average startle amplitude to the silent gap-cued trials divided by the average amplitude of the uncued trials. The startle response ratio $<1$ signifies a silent-gap induced reduction of the startle response. For example, a startle response ratio of 0.6 indicates a $40 \%$ reduction of the startle amplitude for the cued trials. A startle response ratio of 1 suggests that the animal failed to detect the silent gap.

To access an animal's ability to perform an auditory task, separate from its ability to detect a silent gap, the prepulse inhibition (PPI) task was administered in a separate group of mice immediately before and after ( 2 and $10 \mathrm{~d}$ ) NIHL. The physical setup for the PPI task was identical 
to that of the gap detection. However, the trial structure differed in that carrier tone was absent and a white noise burst was cued by a $50 \mathrm{~ms}$ pure tone pulse (frequency pseudorandomly selected from 5, 7, 10, 14, 20, 28, or $45 \mathrm{kHz}$, all at $75 \mathrm{~dB}$ SPL). In short, the PPI task tests an animal's ability to detect a pure tone pulse in silence, while the gap detection task measures an animal's ability to detect a silent gap in a continuous pure tone.

Mice were first acclimated to the testing chamber and trained until the behavior stabilized across $2 \mathrm{~d}$. On average, 1000 trials were given before the first test session. We compared individual animals' performance before and after the experimental manipulation. An increase of gap ratio accompanied by normal ABR for the intact ear and normal PPI behavior were assumed to indicate tinnitus. Because both the gap detection task and the PPI task require normal-hearing and hearing sensitivity was highly variable across animals $>32 \mathrm{kHz}$, only trials with carrier frequencies between 5 and $20 \mathrm{kHz}$ were included in the final analysis.

\section{Lentivirus injection in auditory cortex (AI)}

Posttranscriptional gene silencers were used to manipulate the level of GAD65 expression. A lentivirus carrying an shRNA (Santa Cruz Biotechnology) sequence complementary to mouse GAD65 mRNA was used to knock down GAD65 expression. To account for possible tissue damage from cortical microinjection or viral infection, a lentivirus carrying a non-gene-specific scrambled shRNA sequence (Santa Cruz Biotechnology) was used as a control. Mice were anesthetized with ketamine (100 $\mathrm{mg} / \mathrm{kg}$, IP) and xylazine (10 mg/kg, IP). Injection was done stereotactically to the right AI. A burr hole was made on the temporal ridge $1.75 \mathrm{~mm}$ anterior from the junction between the temporal ridge and the transverse suture. A micropipette filled with the virus solution was lowered down $500 \mu \mathrm{m}$ from the pial surface and $1 \mu \mathrm{l}$ of virus solution was injected at $100 \mathrm{nl} / \mathrm{min}$ by pressure injection (Stoelting Quintessential Injector). The micropipette was then retracted $250 \mu \mathrm{m}$ and an additional $1 \mu \mathrm{l}$ of virus solution was injected. To minimize leaking, the micropipette was left in place for $8 \mathrm{~min}$ before being withdrawn. After injection, the skin was sutured and the animals were returned to their home cages after regaining movement. For postoperative pain management, animals received subcutaneous injection of buprenorphine $(0.05 \mathrm{mg} / \mathrm{kg}, \mathrm{SC})$ and meloxicam $(2 \mathrm{mg} / \mathrm{kg}, \mathrm{SC})$.

\section{Conditioned active avoidance behavioral measure of tinnitus}

In addition to gap detection and PPI, we trained a set of $\operatorname{Tg}(\mathrm{dl}$ x6acre) 1 Mekk mice on a third conditioning-based task-a modified version of an active avoidance task(Yang et al., 2011). This strain was used in active avoidance test because, as our preliminary results indicated, the mice readily learned the task, whereas mice of the other two strains, the FVB in particular, did not Learn the task well. PPI and gap detection depends on a presence of an animal's startle response, which can be affected by a hearing loss (see Lobarinas et al., 2013 and Results). The active avoidance task was used to validate our gap detection results since it uses animal's voluntary movement (i.e., shuttle box chamber crossings) during silent probe trials as an index of presumed tinnitus, and does not rely on acoustic startle response.

Training took place in a two-compartment shuttle box separated by a barrier with a narrow open door connecting the two compartments. The shuttle box was equipped with a grid floor connected to a scrambled shock generator (MED Associates), and was located in a sound attenuation box. Animals were first habituated in the shuttle box for two $1 \mathrm{~h}$ daily sessions, followed by $1-2$ weeks of training in the active avoidance task.

Each daily training session consisted of 60 trials. A total of nine sounds were used in the active avoidance training and tinnitus testing: five pure tones $(4,8,16,20$, and $32 \mathrm{kHz})$ and four octave-band noise $(4-8,8-16$, $10-20$, and $16-32 \mathrm{kHz}$ ) played at four intensities $(30,40,50$, and $60 \mathrm{~dB})$. Due to the small trial numbers in each condition, accuracy was calculated across all frequency-intensity pairs. Each trial started with a holding period of 5 to $40 \mathrm{~s}$, during which the animal had to stay in one side of a two-compartment shuttle box. At the end of the holding period, a sound was played, and the animal had to respond within $7 \mathrm{~s}$ after the sound onset by moving through the small open door into the other side of the shuttle box. If the animal successfully crossed the door within $7 \mathrm{~s}$ of sound onset, the sound was stopped and a new trial was automatically initiated. If the animal failed to cross through the door within the allowed time window, a mild foot shock ( $0.4 \mathrm{~mA}$ for $1 \mathrm{~s})$ was delivered once every $5 \mathrm{~s}$ until the cross was made. The sound was terminated as soon as the animal crossed into the other side of the shuttle box and a new trial was automatically started. False positive trials were aborted, and a new trial was automatically initiated. To facilitate the learning process, false positive crosses during the holding period were not punished during the first few training sessions. After the animal reached a performance level of $50 \%$ avoidance, it received a single foot shock for each false positive cross. The trial was then aborted, and a new trial was automatically initiated. False positive trials were not counted. It took 1-2 weeks for an animal to reach a criterion of $70 \%$ avoidance in three consecutive sessions.

After animals reached the $70 \%$ criterion, they underwent six daily testing sessions. In each testing session, unreinforced probe trials (10 silent and 2 sound probe trials, both $1 \mathrm{~min}$ long) were introduced and randomly mixed with 48 reinforced trials. Because animals tended to cross fewer times in silent probe trials, we included more silent than sound probe trials (10 to 2 ) to obtain more accurate measurements. Frequency and intensity of the sound probe trial was randomly chosen from those used in the training. To avoid extinction of learned responses, we only included a small number (12) of these unreinforced probe trials in each test session. A sound was played during the entire 1 min duration of the sound probe trial, whereas no sound was played during the entire 1 min duration of the silent probe trial. Animals were allowed to either stay in one compartment or cross into the other side freely during these probe trials. Due to a lack of feedback, such as the foot shock upon incorrect crossing or the termination of sound upon correct crossing, mice tended to make multiple crosses during the 1 min probe trials. The total number of crosses in each trial, and not the binary presence or absence of the crosses per trial, was recorded. Six test sessions were conducted per animal, yielding 60 silent and 12 sound probe trials.

The number of crosses in silent probe trials measures the likelihood that the animal was hearing tinnitus, but might also be influenced by the animal's motivation and motor activity, which were assessed with the number of crosses in the sound probe trials. To control for these factors, the average per-trial-crosses of the silent probe trials were divided by the average per-trial-crosses of the sound probe trials at the end of each testing session. The baseline behavioral score was calculated by taking an average of this silent-over-sound cross ratio across six sessions.

Once the baseline behaviors were recorded, animals received either monaural NIHL or a cortical microinjection of GAD65 shRNA. Fifteen days later, animals received another $6 \mathrm{~d}$ of tinnitus testing, using the same procedure as described before. An increase of the silent-over-sound cross ratio after NIHL or GAD65 knock-down was considered evidence of tinnitus perception.

\section{Measuring GAD65 mRNA levels with RT-PCR}

After behavioral testing, animals were euthanized with isoflurane. Brain tissue was collected from the right and left auditory cortices based on anatomical landmarks by an experienced experimenter (S.Y.). A coronal slice of $\sim 1 \mathrm{~mm}$ thickness (estimated stereotaxic coordinates: $-2 \mathrm{~mm}$ to $-3 \mathrm{~mm}$ bregma) was made using the dorsal-ventral extent of the hippocampus as landmarks. We then hemisected and isolated the AI at each side by making two orthogonal cuts to the cortical surface at 1 and $2 \mathrm{~mm}$ dorsal to the lingual gyrus. Subcortical structures were removed and two $1 \mathrm{~mm}$ cubes of cortical tissue, one from each side, were collected. These samples presumably included the primary AI and possibly other fields of the AI.

RT-PCR was conducted by an experimenter (S.J.C.) who was blinded to the experimental conditions. Total RNA samples were prepared from the tissue with RNA Wiz (Ambion) according to the manufacturer's instructions. The total RNA obtained $(\sim 3 \mu \mathrm{g})$ was reverse-transcribed using a first-strand cDNA synthesis kit (BD Biosciences). The PCR mixture $(50 \mu \mathrm{l})$ contained $10 \times$ Taq buffer, $0.3 \mathrm{U}$ Taq polymerase (PerkinElmer), $2.5 \mu \mathrm{M}$ dNTPs, 5 pmol of each set of primers, and $50 \mathrm{ng}$ of cDNA from the AI as template. GAD65-specific fragments were amplified with the following PCR primers: GAD65-F: 5'-GCGCAGTTCTTGCTGGAA GTGGTAGACATA-3', GAD65-R: 5'-AGGGTTCCAGGTGACTGAAT TGGCCCTTTC-3'. PCRs were performed under the following cycling 
conditions: an initial denaturation at $94^{\circ} \mathrm{C}$ for $5 \mathrm{~min}$ followed by $25-40$ cycles of denaturation at $94^{\circ} \mathrm{C}$ for $30 \mathrm{~s}$, annealing at $63^{\circ} \mathrm{C}$ for $30 \mathrm{~s}$, and elongation at $72^{\circ} \mathrm{C}$ for $1 \mathrm{~min}$ with a final elongation step at $72^{\circ} \mathrm{C}$ for 10 min. A $10 \mu \mathrm{l}$ sample of each PCR was removed after 25 cycles, while the remaining mixture underwent 5 more cycles of amplification. The extent of amplification was chosen empirically to avoid saturation of the amplified bands. In addition, two samples were collected for optimal quantification of GAD65 expression levels. Each primer set yielded a PCR product of $870 \mathrm{bp}$ in length for GAD65. The 18S rRNA gene was used as an internal standard (QuantumRNA, Ambion). To quantify PCR products, each sample was run in a $1.5 \%$ agarose gel and stained with ethidium bromide. Band intensity was measured with an Alphaimager (Alpha Innotech) using the Alphaease (v3.3b) program.

\section{AI mapping and recording of spontaneous and evoked multiunit activity}

The AI of each animal was mapped 20-30 d after monaural NIHL as described above, or virus injection (C57BL/6 NIHL $(n=5), 20 \pm 2 \mathrm{~d}$ postprocedure; FVB NIHL $(n=4), 26 \pm 4$ d; FVB GAD65 $\operatorname{shRNA}(n=$ $6), 23 \pm 3 \mathrm{~d}$; FVB scrambled shRNA $(n=5), 28 \pm 1 \mathrm{~d})$.

Animals were anesthetized with ketamine $(100 \mathrm{mg} / \mathrm{kg}$, IP) and xylazine $(10 \mathrm{mg} / \mathrm{kg}, \mathrm{IP})$, and maintained at $36.5^{\circ} \mathrm{C}$ with a homeothermic heating pad (Harvard Apparatus). The anesthetized mouse was placed in a custom head holder and a slit was made in the cisterna magna to drain CSF and reduce brain pulsations. A craniotomy and durectomy was performed over AI. A layer of silicon oil was applied to prevent brain desiccation. Sound stimuli were generated by an audio signal processor (Tucker-Davis Technologies RX6) and delivered to the ear contralateral to the craniotomy, through an enclosed cannulated speaker (TuckerDavis Technologies Electrostatic Speaker, Coupler model). The speaker was calibrated to have $<3 \%$ harmonic distortion and flat output in the entire frequency range (Tucker-Davis Technologies SigCal32).

Multiunit responses of AI neurons were recorded using tungsten microelectrodes (FHC) advanced orthogonally to the cortical surface to approximately cortical layer IV $(350-400 \mu \mathrm{m})$. Electrical signals were amplified and recorded for each 333 ms trial (Tucker-Davis Technologies RX5). Before each recording block, search stimuli (white noise bursts, $60 \mathrm{~dB}$ SPL, $25 \mathrm{~ms}$ duration, repeated at $3 \mathrm{~Hz}$ ) were played to identify sound-evoked multiunit responses. Multiunit responses were defined as voltage changes that exceeded the mean amplitude of the baseline electrical trace by two SDs. Thresholds for multiunit discrimination were set for each microelectrode before each recording block. For each recording block of $6 \mathrm{~min}$, pure tone pips of 51 frequencies were presented in pseudorandom order $(4-64 \mathrm{kHz}, 0.1$ octave spacing, $5 \mathrm{~ms}$ cosine-squared ramps, $25 \mathrm{~ms}$ duration, repeated three times) at 8 intensities (0-70 dB SPL, $10 \mathrm{~dB}$ spacing), starting at $150 \mathrm{~ms}$ into the trial. Multiunit responses were used to reconstruct the frequency-intensity receptive field of each recording site and to calculate the tone evoked firing rate. To avoid a frequency sampling edge at 4 and $64 \mathrm{kHz}$, only units with characteristic frequencies (CFs) between 8 and $32 \mathrm{kHz}$ were included in the analyses.

Following the sound presentation block, multiunit spontaneous activity was recorded while maintaining identical electrode location and threshold. We measured the spontaneous rate in a separate 5 min silent block, rather than the interstimulus periods in the sound presentation block, to minimize the influence of recent $(<333 \mathrm{~ms})$ sound presentation on multiunit activity. Multiunit spike threshold was set at 2 SDs above and below the noise envelope for all experiments and fixed throughout the recording for each unit. The tone evoked firing rate was defined as the mean firing within the full duration at half maximum of each unit's peristimulus time histogram (PSTH) after subtracting the spontaneous firing rate. Spontaneous firing rate was calculated as the mean firing for the entire duration ( $5 \mathrm{~min}$ ) of the silent trials.

The location and boundaries of AI were identified based on a combination of stereotaxic coordinates, vascular landmarks, and response properties of the multiunit responses. Multiunits in AI typically exhibit short response latencies, a sharp peak in PSTH, reliable responses to rapidly successive $(3 \mathrm{~Hz})$ tonal stimulation, and caudal-to-rostral tonotopic frequency gradient. In case the tonotopic gradient was disrupted by noise exposure, the low-frequency-selective units in the caudal side of the AI were used to mark the caudal end of AI, and more rostral areas were carefully searched for AI units. Electrode penetrations were made densely and evenly throughout AI while avoiding surface blood vessels. Recording sites were marked on a magnified digital photograph of the cortex for later tonotopic map reconstruction.

After recordings were made on one side of the cortex, the craniotomy was covered with additional silicone grease and was sealed with a plastic film, and the mapping procedures were repeated on the other side. Surgery order for the two cortical sides was pseudorandomized across animals. All animals included in the cortical mapping experiment underwent bilateral AI mapping, and were euthanized at the end of mapping.

\section{Characterization of receptive field properties}

Frequency-intensity receptive fields (RFs) of each multiunit were constructed from the peak firing period of the PSTH. The peak firing period was defined as the full duration at half maximum of the PSTH after subtracting the spontaneous firing rate. The frequency response area (FRA) was defined as the region within the frequency-amplitude space where firing rate exceeded $1 / 4$ of the multiunit's peak firing. The size of FRA was defined as the number of tones (i.e., frequency-intensity combinations) in the FRA. The firing threshold was calculated as the lowest sound intensity at which suprathreshold firing was evoked in the FRA. The characteristic frequency was the center frequency bin at the firing threshold, and corresponds to the tip of the V-shaped FRA. Thresholds and CFs are determined by custom MATLAB program confirmed by visual inspection. Auditory cortical tonotopic map was reconstructed by Voronoi tessellation (MATLAB, The MathWorks).

\section{Experimental design and statistics}

Statistical analyses were conducted using MATLAB and SPSS (IBM). Repeated-measures ANOVAs followed by post hoc $t$ tests were conducted to evaluate the statistical significance of the differences between groups or experimental procedures. Analysis of covariance (ANCOVA) was used to examine multiunit threshold differences between groups, using the CF as the covariant. Linear correlation between two variables was tested by Pearson correlation. Two-tailed tests were used unless otherwise stated. The significance level was set at $\alpha=5 \%$. Data are presented as mean \pm SEM unless otherwise stated.

\section{Results}

\section{Strain difference in hearing loss-induced tinnitus}

Several studies demonstrated noise exposure-induced tinnitus in CBA/Caj mice (Longenecker and Galazyuk, 2011; Middleton et al., 2011; Llano et al., 2012). To assess whether NIHL results in tinnitus in FVB and C57BL/6 mice, we exposed the left ear of the mice to a $112-\mathrm{dB} 8 \mathrm{kHz}$ tone for $2 \mathrm{~h}$ and tested gap detection behavior (Fig. 1a) before and after the exposure. Noise exposure resulted in significant and persistent $\mathrm{ABR}$ threshold shifts for the exposed (left) ear in both C57BL/6 $(n=8)$ and FVB mice $(n=8)$ (ANOVA, session, $F_{(1,14)}=374.64, p<0.001$, paired-samples $t$ test, $p<0.05$ for $4-32 \mathrm{kHz}$; Fig. $1 b, c$, top). This ABR threshold shift was maintained for at least $20 \mathrm{~d}$ after NIHL. The magnitudes of the ABR threshold shifts were similar between the two strains (ANOVA, strain-by-session interaction, $F_{(1,14)}=1.02, p=0.33$ ). In both strains, the protected (right) ear maintained preexposure threshold (Fig. 1b,c, top), peak latency (ANOVA, session $F_{(1,12)}=0.51, p=0.49$; session-by-strain interaction, $F_{(1,12)}=$ $0.42, p=0.53$ ), and peak amplitude (ANOVA, session, $F_{(1,13)}=$ $0.07, p=0.79$; session-by-strain interaction, $F_{(1,13)}=0.28$. $p=0.61$ ), indicating a successful monaural NIHL. Hearing in the protected ear allowed the mice to perform in subsequent gap detection, prepulse inhibition and active avoidance tests.

Tinnitus was measured by the impairment it causes in detecting a gap in a constant background sound (Turner et al., 2006; Fournier and Hébert, 2013). Gap detection was quantified by the 
a
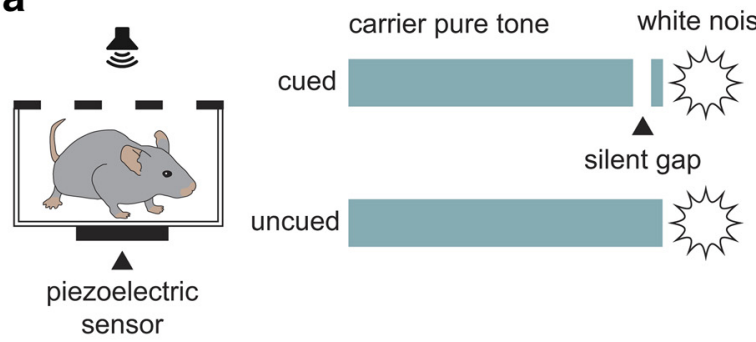

b

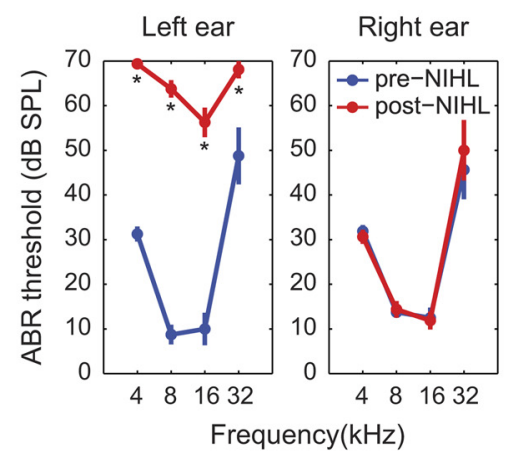

C57BL/6 gap detection

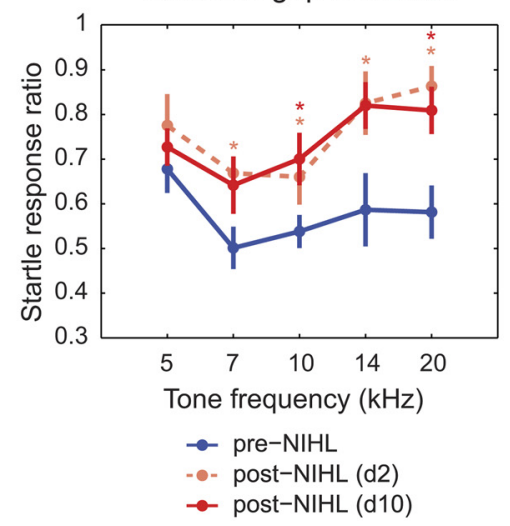

d

Individual mean change

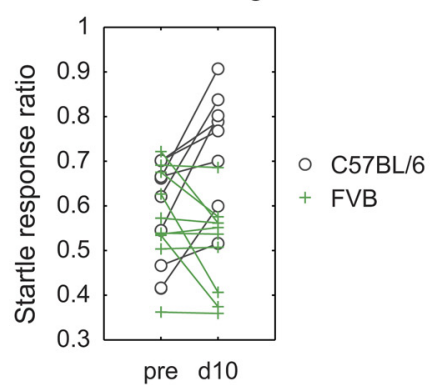

C

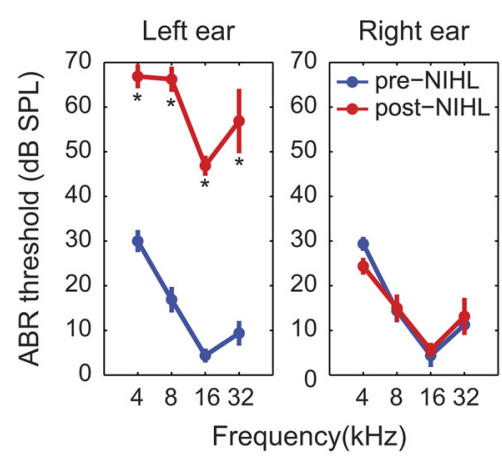

FVB gap detection

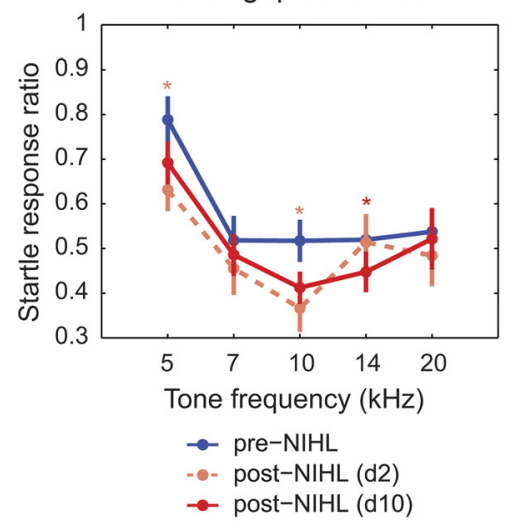

e Post-NIHL GAD65 mRNA

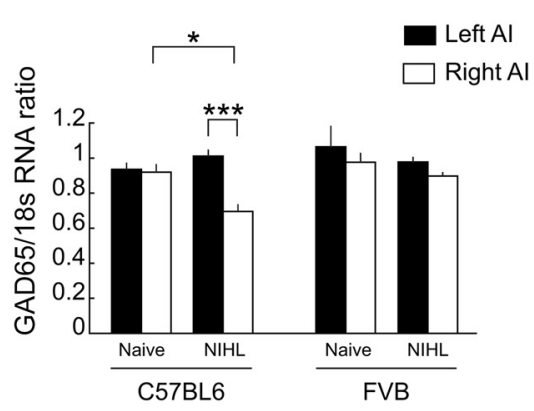

Figure 1. Strain differences in gap detection and cortical GAD65 level following NIHL. $\boldsymbol{a}$, Schematic representation of the gap detection setup (left), sound stimulus (middle), and average startle response trace for cued and uncued trials (right). (b-c, top) Auditory brainstem response thresholds before and $15-20 \mathrm{~d}$ after monaural NIHL to the left ear in $\boldsymbol{b}, \mathrm{C} 57 \mathrm{BL} / 6$ and $\boldsymbol{c}$, FVB mice. $\boldsymbol{b}$, c, Bottom, Gap detection behavior before, $2 \mathrm{~d}(\mathrm{~d} 2)$ after, and $10 \mathrm{~d}(\mathrm{~d} 10)$ after NIHL in $\boldsymbol{b}, \mathrm{C57BL} / 6$ and c, FVB mice. Statistical significance was determined by ANOVA followed by a post hoc paired $t$ test ${ }^{*} p<0.05$. $\boldsymbol{d}$, Individual animals' average (tone frequency-collapsed) gap detection behavior for before and $10 \mathrm{~d}$ after NIHL. Line color indicates the mouse strain used (gray = C57BL/6, green = FVB). $\boldsymbol{e}$, AI GAD65 mRNA level for C57BL/6 and FVB mice $10 \mathrm{~d}$ after NIHL as measured by the ratio of GAD65 to 18s RNA. Bar color indicates the cortical side (white is right/AI contralateral to noise-exposed ear, black is left/Al ipsilateral to noise-exposed ear, in the same animal). ${ }^{*} p<0.05 ;{ }^{* *} p<0.001$. Error bars represent \pm SEM.

ratio of cued-over-uncued average startle amplitudes, with a lower ratio indicating better gap detection. Gap detection was examined in three sessions-pre-NIHL, and 2 and $10 \mathrm{~d}$ post-NIHL-in FVB $(n=$ $10)$ and $\mathrm{C} 57 \mathrm{BL} / 6$ mice $(n=8$; Fig. $1 b, c$, bottom). Before NIHL, FVB mice exhibited higher raw startle amplitude than C57BL/ 6 for both cued and uncued trials $(5.91 \pm 0.44 \mathrm{~V}$ of amplified signal for FVB and $3.11 \pm 0.61 \mathrm{~V}$ for $\mathrm{C} 57 \mathrm{BL} / 6 ; p<0.05$, unpaired $t$ test). However, there was no strain difference in gap detection performance as measured by startle response ratio (i.e., cued-over-uncued average startle amplitudes ratio) for any carrier tone frequency (ANOVA, strain-by-frequency interaction, $F_{(4,14)}=1.93, p=0.16$; Fig. 1$)$. After NIHL, we observed a general reduction in raw startle amplitude in both strains $(37.1 \pm 8.1 \%$ for FVB and $36.6 \pm$ $17.6 \%$ for C57BL/6; $p<0.05$ for both strains, paired $t$ test). The percentage reduction of uncued raw startle amplitude was similar between the two strains (ANOVA, session-by-strain interaction, $\left.F_{(1,16)}=0.07, p=0.80\right)$. Reduction of acoustic startle reflex after NIHL has previously been reported, and raised a concern that it could bias for a higher startle response ratio in gap detection (Lobarinas et al., 2013). However, there was no correlation between raw startle amplitude and the startle response ratio in our study (Pearson correlation between average uncued startle amplitude and associated startle response ratio. $r=0.04, n=180$, $p=0.64$. Data pooled from all subjects and test sessions).

Interestingly, NIHL had different effects on the gap detection in the two strains of mice as indicated by a significant strain-by-session interaction (ANOVA, $\left.F_{(2,14)}=13.98, p<0.001\right)$. Although the gap detection performance was similar across two strains before NIHL, strain differences emerged after NIHL, with C57BL/6 showing worse gap detection than FVB mice $(p<0.01$ for $7,10,14$, and $20 \mathrm{kHz}$ at day 2 and $p<0.01$ for 10,14 and $20 \mathrm{kHz}$ at day 10 , unpaired $t$ test; see Fig. 1 , $b$ and $c$ for performances). A comparison of each individual animal's behaviors preand post-NIHL confirmed that while NIHL significantly impaired gap detection in C57BL/6 (Fig. 1b, bottom, $d$ : paired $t$ test, $p<0.05$ for $7,10,14$, and 20 $\mathrm{kHz}$ at $\mathrm{d} 2$ and $p<0.05$ for 10 and $20 \mathrm{kHz}$ at $\mathrm{d} 10$ ), it improved gap detection in FVB mice (Fig. $1 c$, bottom, $d$ : paired $t$ test, $p<$ 0.05 for $5 \mathrm{kHz}$ and $10 \mathrm{kHz}$ at $\mathrm{d} 2$ and $p<$ 0.05 for $14 \mathrm{kHz}$ at d10). These results suggest that hearing in the protected right ear 
was sufficient for the gap detection test, and the impairment in gap detection in the C57BL/6 mice was likely due to tinnitus induced by noise exposure to the left ear.

To further confirm that impairment of gap detection indicates tinnitus, not hearing impairments, we tested prepulse inhibition (PPI) behavior in a separate group of C57BL/6 $(n=7)$ and FVB $(n=7)$ mice after the same NIHL procedure as the previous experiment (see Materials and Methods for difference between gap detection and PPI task). NIHL did not affect PPI in either $\mathrm{C} 57 \mathrm{BL} / 6$ or FVB mice (ANOVA, session, $F_{(2,11)}=1.58, p=$ $0.25)$. In addition, no strain difference was observed in PPI (ANOVA, session-bystrain interaction, $\left.F_{(2,11)}=2.7, p=0.11\right)$. These results suggest that $\mathrm{C} 57 \mathrm{BL} / 6$ and FVB mice with monaural NIHL could hear the prepulse tones similarly well with the protected ear.

This specific impairment of gap detection in $\mathrm{C} 57 \mathrm{BL} / 6$, but not in FVB mice, suggest that $\mathrm{C} 57 \mathrm{BL} / 6$ mice are susceptible to hearing loss-induced tinnitus, whereas FVB mice do not develop tinnitus with the specific sound exposure paradigm used here.

\section{Strain differences in cortical GAD65 expression after NIHL}

Previous studies have implicated GAD65 regulation in tinnitus (Yang et al., 2011). To determine whether the strain difference in tinnitus susceptibility is related to differential modulation of GAD65 by NIHL, we quantified its expression in the AI of mice after the tinnitus test. Brain tissue samples were taken from the left and right (ipsilateral and contralateral to the noise-exposed ear) auditory cortices of C57BL/6 (NIHL: $n=10$, naive: $n=5$ ) and FVB mice (NIHL: $n=7$, naive $n=4$ ). The level of GAD65 mRNA was measured by RT-PCR.

After NIHL, C57BL6 mice showed a reduction of GAD65 mRNA in the (right) cortex contralateral to the noise exposed ear $\left(t_{13}=0.37, p=0.04\right)$ and an increase in the ipsilateral (left) cortex $\left(t_{13}=2.80\right.$, $p=0.01)$ compared with naive condition. Each C57BL/6 mice also showed a lower GAD65 level in right cortex compared with its left cortex after NIHL $\left(t_{9}=5.25\right.$ $p=0.0005)$. FVB mice did not show changes in GAD65 mRNA level post NIHL (Fig. 1e). The lack of hearing lossinduced GAD65 downregulation in FVB mice indicates that they may be impaired in this particular form of homeostatic plasticity. Some other inbred strains have

\section{a Posttranscriptional gene knockdown using lentivirus shRNA particles}

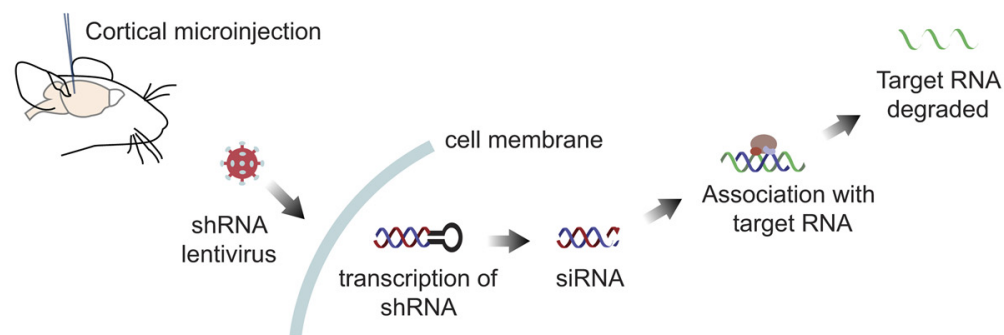

b

FVB GAD65 shRNA ABR

C

FVB scrambled shRNA ABR
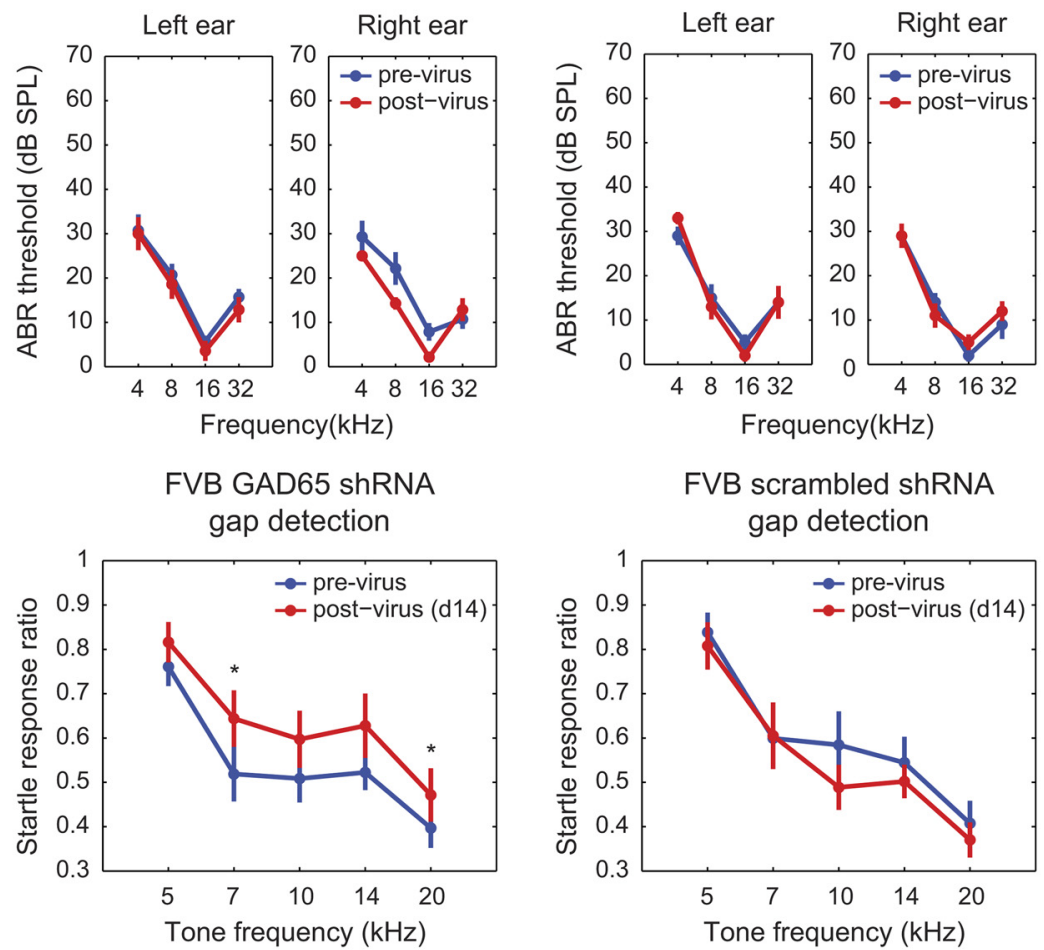

d

Individual mean change

e FVB post-virus injection GAD65 mRNA
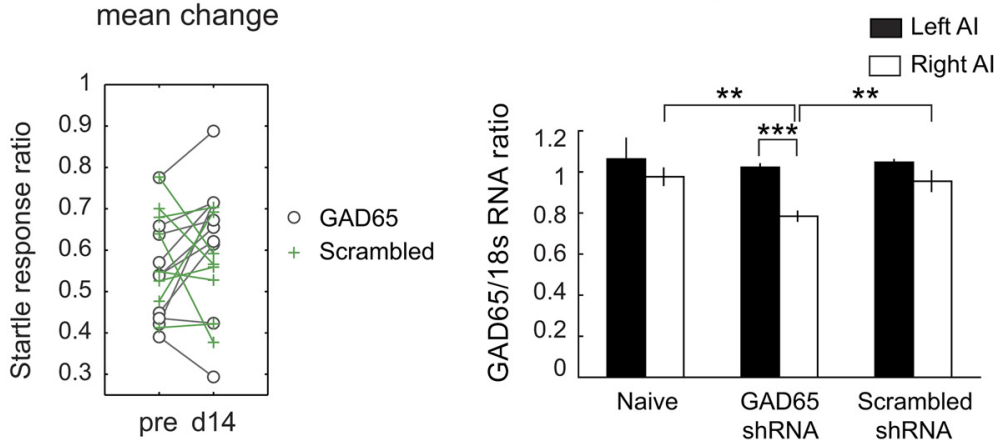

Figure 2. Cortical GAD65 knock-down in FVB mice is sufficient to induce tinnitus. $\boldsymbol{a}$, Schematic representation of cortical GAD65 knock-down using shRNA lentiviral particles. $\boldsymbol{b}, \boldsymbol{c}$, Top, Auditory brainstem response thresholds before and 15-20 $\mathrm{d}$ after injection of GAD65 shRNA (b) and control scrambled shRNA (c) to the right Al. Bottom, Gap detection behavior before and $14 \mathrm{~d}$ (d14) after injection of $\boldsymbol{b}$, GAD65 shRNA and $\boldsymbol{c}$, control scrambled shRNA. Statistical significance was determined by ANOVA followed by post hoc paired $t$ test ${ }^{*} p<0.05$. $\boldsymbol{d}$, Individual animal's average (tone frequency-collapsed) gap detection behavior for before and $14 \mathrm{~d}$ after shRNA injection. Line color indicates the virus type used (gray is GAD65 shRNA, green is control scrambled shRNA). $\boldsymbol{e}$, AI GAD65 mRNA level $14 \mathrm{~d}$ after shRNA injection as measured by the ratio of GAD65 to 18s RNA. Bar color indicates the cortical side (white is right/shRNA injected Al, black is left/uninjected Al, in the same animal). ${ }^{* *} p<0.01 ;{ }^{* *} p<0.001$. Error bars represent \pm SEM. 
also been shown to be impaired in homeostatic synaptic plasticity in the neocortex (Ranson et al., 2012).

Comparing the C57BL/6 and FVB populations, the significance in strain difference (ANOVA, side-by-strain interaction $\left.F_{(1,15)}=9.34, p=0.008\right)$ is driven by the reduction of GAD65 level in the right cortex of C57BL6 compared with FVB mice $\left(t_{15}=2.71, p=0.02\right.$, left cortex n.s.). Together with strain specific impairment of gap detection in $\mathrm{C} 57 \mathrm{BL} / 6$, we hypothesized that post-NIHL GAD65 downregulation may be a factor contributing to tinnitus behavior.

\section{Induction of tinnitus in FVB mice by GAD65 knock-down}

To test whether cortical GAD65 knock-down alone is sufficient to induce tinnitus, we performed a posttranscriptional knock-down of GAD65 expression using a small hairpin RNA (shRNA) (Fig. 2a). GAD65 knock-down experiments were performed on normal-hearing FVB mice that had never undergone NIHL. Tinnitus symptoms were identified using a gap detection task. A second GAD65 knock-down experiment, described later, validates the results using an additional mouse strain and an alternative behavioral task.

Lentivirus particles carrying GAD65 shRNA were injected in the right AI of the FVB mice $(n=14)$. Control FVB mice $(n=10)$ underwent identical surgical procedures and were injected with lentivirus particles carrying shRNA of non-gene-specific scrambled sequences. Gap detection or PPI tests was administered in two sessions: preinjection and $14 \mathrm{~d}$ post-injection. ABRs were examined 15-20 d after injection in a subset of the mice that underwent behavioral tests.

Virus injection and cortical GAD65 knock-down did not alter the ABR threshold (Fig. 2b,c, top), peak latency (ANOVA, session $F_{(1,10)}=0.36, p=0.56$; session-by-virus type interaction, $F_{(1,10)}=$ 2.34, $p=0.16$; session-by-virus type-by-ear interaction, $F_{(1,10)}=$ $0.65, p=0.44$ ) or peak amplitude (ANOVA, session $F_{(1,10)}=0.37$, $p=0.65$; session-by-virus type interaction, $F_{(1,10)}=7.73, p=$ 0.06 ; session-by-virus type-by-ear interaction, $F_{(1,10)}=1.16, p=$ 0.31 ) in signals recorded from left or right ear after shRNA injection (GAD65 knock-down, $n=7$; scrambled sequence, $n=5$ ). The startle amplitude for uncued trials did not differ between preinjection and postinjection sessions, or between the GAD65 knock-down and the scrambled control groups $(p>$ 0.19 for all frequencies, paired and unpaired $t$ tests, respectively). Gap detection, as measured by the cued-over-uncued startle response ratio, was similar across groups before the injection (ANOVA, virus type-by-frequency interaction, $\left.F_{(4,13)}=0.29, p=0.88\right)$. However, it was significantly impaired by GAD65 knock-down but not by the injection of the scrambled sequences (Fig. 2b,c, bottom, $d$ : ANOVA, sessionby-virus type interaction $\left.F_{(1,16)}=4.64, p=0.047\right)$. Post hoc paired $t$ test confirmed the impaired gap detection in the GAD65 knock-down group (Fig. 2b, bottom: $p<0.05$ for $7 \mathrm{kHz}$ and 20 $\mathrm{kHz}$ ). By contrast, PPI behavior was unaffected in both groups following the virus injection (ANOVA, session, $F_{(1,5)}=5.156, p=0.07$, session-by-virus type interaction, $\left.F_{(1,5)}=0.041, p=0.848\right)$ confirming that GAD65 knock-down resulted in a task specific deficit in gap detection, but not in PPI.

The degree of GAD65 knock-down was verified in a subset of the mice (FVB naive as control, $n=4$, FVB GAD65 shRNA, $n=8$; FVB scrambled shRNA, $n=5$ ) after the tinnitus test. Significantly greater reduction of GAD65 mRNA was confirmed in the injected side of the GAD65-knock-down group (Fig. 2e: group $\times$ cortical side 2-way ANOVA, group interaction $\left.F_{(2,28)}=4.798, p=0.016\right)$. Post hoc LSD tests indicated

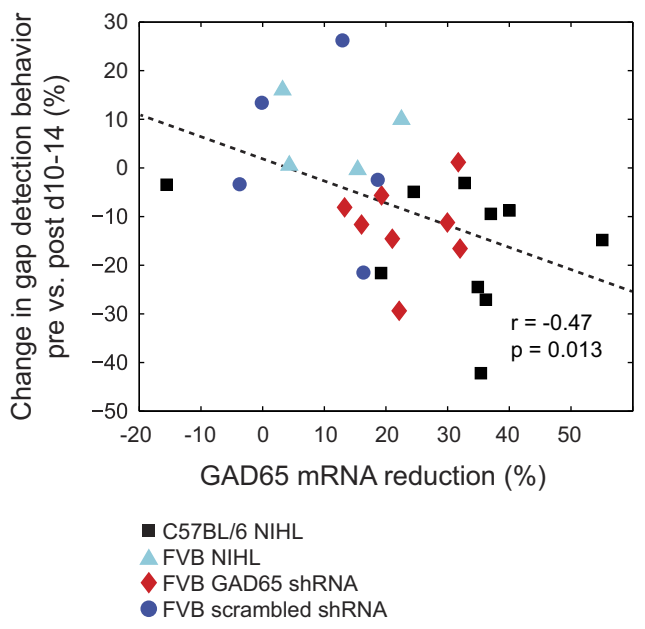

Figure 3. Cortical GAD65 reduction correlates with severity of tinnitus. Tinnitus behavior is significantly correlated with reduction in cortical GAD65 mRNA level. The $x$-axis shows the percentage of GAD65 mRNA reduction in the treated/right cortex relative to the intact/left cortex. The $y$-axis shows the percentage change in gap detection behavior before and after the experimental manipulation (i.e., NIHL or shRNA injection). Postmanipulation behavior was assessed at d10 in NIHL case and d14 in shRNA case. Zero-value indicates no change, positive value indicates improvement and negative value indicates impairment. One-tailed Pearson correlation $(r=-0.47, n=27, p=0.013)$.

that the left/right different occurred in the shRNA group but not the control and scrambled groups, and that the GAD65 mRNA level was significantly lower in the shRNA group than the control and scrambled groups, but only for the right side (Fig. 2e). We also found similar levels of GAD65 mRNA in the right $\mathrm{AI}$ of C57BL/ 6 mice with NIHL compared with FVB mice with shRNA treatment (unpaired $t$ test: $t_{16}=0.885, p=0.40$ ), indicating that NIHL and GAD65 shRNA injection procedures resulted in similar levels of GAD65 reduction. Together, these results show that artificial GAD65 knock-down in the AI is sufficient to induce tinnitus in FVB mice with normalhearing.

\section{Correlation between tinnitus and cortical GAD65 reduction}

We observed both individual and strain differences in the behavioral measure of tinnitus and the downregulation of cortical GAD65 expression, in both virus-injected and noiseexposed mice. To test whether there was an overall correlation between tinnitus and cortical GAD65 regulation, we pooled together data from all the mice with both measures (C57BL/6 NIHL: $n=10$, FVB NIHL: $n=4$, FVB GAD65 shRNA: $n=8$, FVB scrambled shRNA: $n=5)$. We found a significant linear correlation between the mean behavior change and GAD65 mRNA reduction in the affected (right) cortex (Fig. 3, onetailed Pearson correlation, $r=-0.47, n=27, p=0.013)$. A similar correlation was found for the active avoidance task (Fig. $4 d$, and next section).

\section{Behavioral evidence of tinnitus measured with an active avoidance task}

Although the gap detection method has been widely used to quantify tinnitus, there are lingering questions as to whether performance on it is influenced by processes unrelated to tinnitus (see Discussion). To validate our behavioral results, we measured putative tinnitus using a conditioning-based procedure adapted from a previous report (Yang et al., 2011). Tg(dlx6a-cre)1Mekk 
mice were used for this experiment. We found that, like $\mathrm{C} 57 \mathrm{BL} / 6$, this mouse strain showed a 43\% reduction in GAD65 mRNA level $21 \mathrm{~d}$ after noise exposure ( $n=5$ for the control group and $n=5$ for the noise-exposed group; ANOVA, $F_{(1,14)}=59.2, p<0.01$, post hoc independent $t$ test, $t_{8}=23.84, p=1.83 \times 10^{-6}$ ).

Mice were trained to cross between the two compartments of a shuttle box when a tone was played (Fig. 4a). Crossing behavior during silent probe trials was false alarms presumably triggered by tinnitus (Fig. $4 b$ ). The average number of crosses during silent probe trials was normalized to the average number of crosses during sound probe trials to account for individual differences in motivation and motor activity (see Materials and Methods for details). Training was performed until animals reach a criterion of $70 \%$ accuracy, which took up to 2 weeks. Performance was maintained throughout the duration of all test sessions.

An increase in false alarms was observed after NIHL, supporting the interpretation of impaired gap detection as a symptom of tinnitus. After NIHL, mice showed more crossing during silent periods (one-tailed paired $t$ test, $\mathrm{t}_{4}=-3.53$, $p=0.01)$ and a significant increase in tinnitus score (i.e., ratio of silent-over-sound crossing) (Fig. 4c, NIHL, one-tailed paired $t$ test, $\left.t_{4}=-2.95, p=0.02\right)$.

GAD65 knock-down-mediated tinnitus confirmed by the active avoidance task

To validate GAD65 knock-down-mediated tinnitus with the active avoidance task, we conducted GAD65 knock-down in the right $\mathrm{AI}$ in $10 \mathrm{Tg}$ (dlx6a-cre) 1 Mekk mice with normal-hearing, and compared behavioral evidence of tinnitus before and 15-20 d after the virus injection. We did not use FVB mice because they could not learn the task to the criterion. We aimed for a GAD65 knock-down approximately equivalent to the level that was observed in noise-exposed mice (approx. 40\%). Therefore, we only included mice with $>40 \%$ GAD65 knock-down in AI $(n=6)$ in this analysis. Mice with GAD65 knockdown showed a similar tendency of increased crossing during the silent periods (one-tailed paired $t$ test, $t_{5}=-4.01, p<$ 0.01 ) and increased tinnitus score (Fig. $4 c$, GAD65 knock-down, one-tailed paired $t$ test, $\left.t_{5}=-4.87 p=0.002\right)$, suggesting they were perceiving tinnitus.

We also analyzed whether the amount of GAD65 downregulation correlates with the severity of tinnitus by including all animals that underwent GAD65 shRNA injection and behavioral testing $(n=10)$. We found that GAD65 knock-down was highly

a Training
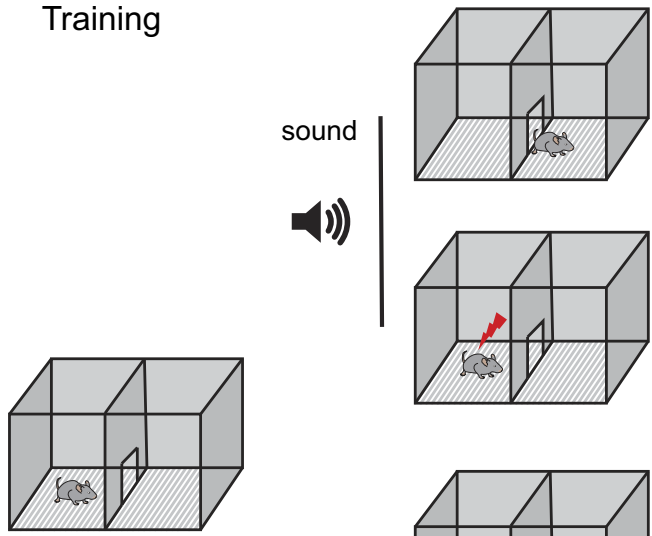

Cross within $7 \mathrm{sec}$ (correct)

sound terminates

sound

$4(4))$

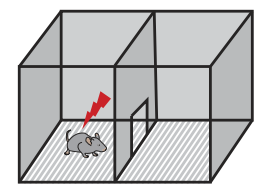

No Cross within $7 \mathrm{sec}$ (incorrect)

foot shocks until the mouse crosses

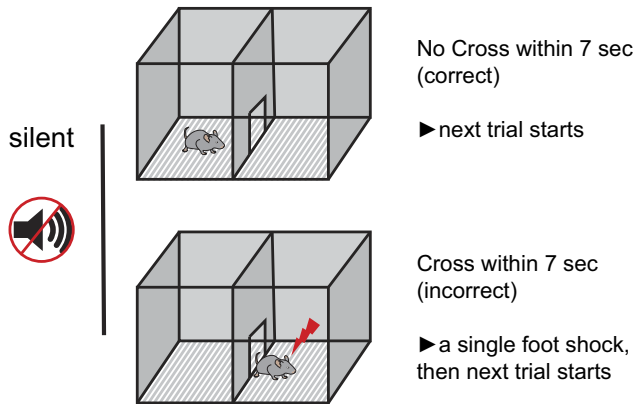

b Test

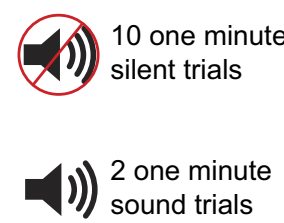

C
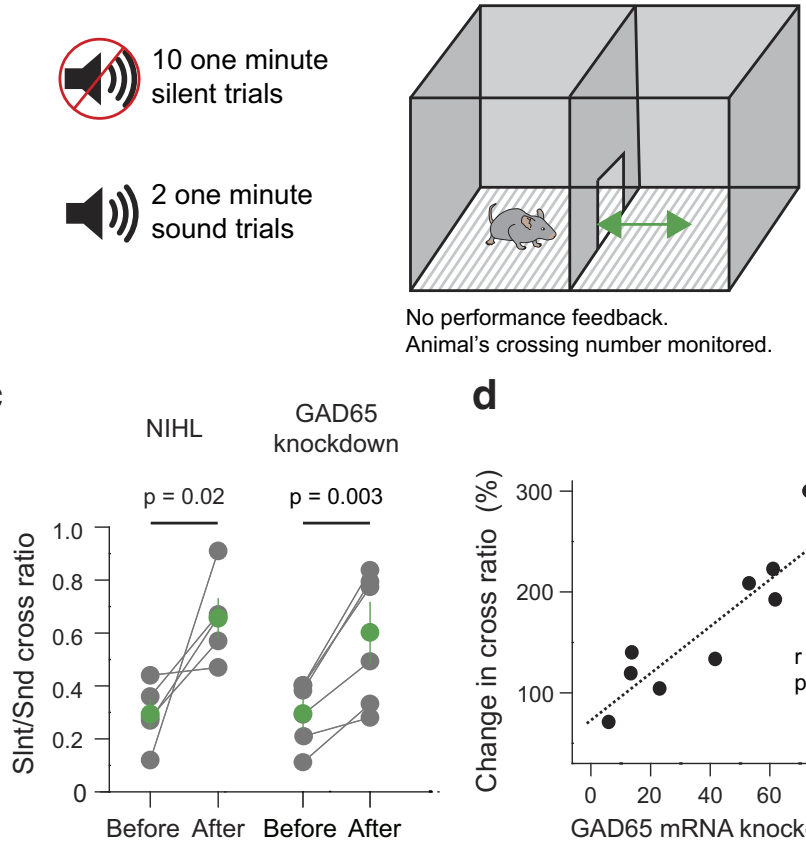

No performance feedback. Animal's crossing number monitored.

d

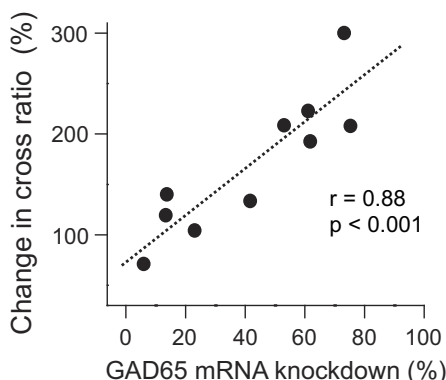

Figure 4. Cortical GAD65 reduction correlates with tinnitus as measured by a conditioned active avoidance task. $\boldsymbol{a}$, Schematic representation of the active avoidance task training. Animals were trained on the reinforced trials until they reach $70 \%$ overall accuracy. $\boldsymbol{b}$, Schematic representation of the active avoidance probe (test) trials. 12 unreinforced probe trials were randomly intermixed with 48 reinforced trials in a test session. c, Tinnitus behavior before and after NIHL or cortical GAD65 knock-down (gray indicates individual mouse, green indicates group mean of the condition). $\boldsymbol{d}$, Tinnitus behavior is significantly correlated with cortical GAD65 mRNA knock-down level (one-tailed Pearson correlation, $r=0.88, n=10, p<0.001$ ). The $x$-axis shows the percentage of GAD65 mRNA reduction in the injected/right cortex relative to the uninjected/left cortex. The $y$-axis shows the percentage change in tinnitus behavior between before and after GAD65 knock-down. correlated with tinnitus quantified using our active avoidance task (one-tailed Pearson correlation, $r=0.88, n=10, p<0.001$; Fig. $4 d$ ). These results are consistent with results obtained with the gap detection, and strengthen our conclusion that downregulation of GAD65 in the $\mathrm{AI}$ is correlated with tinnitus in animal models. 


\section{a Al tonotopy}
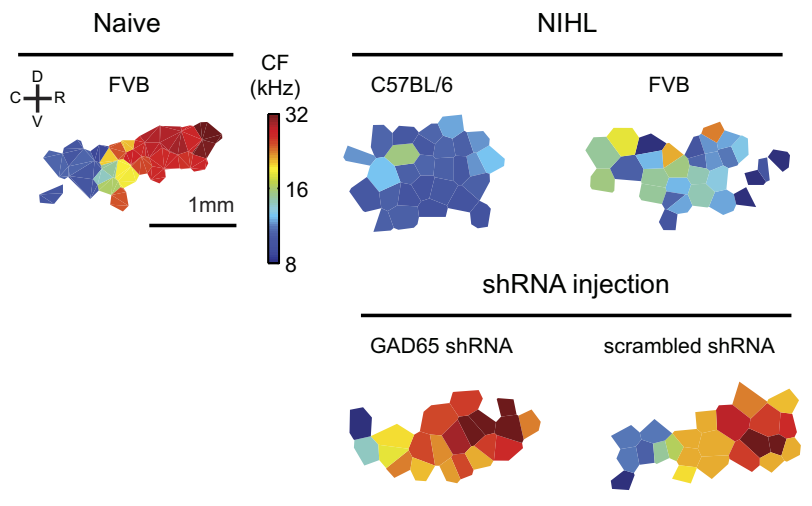

\section{b Tonotopic axis}

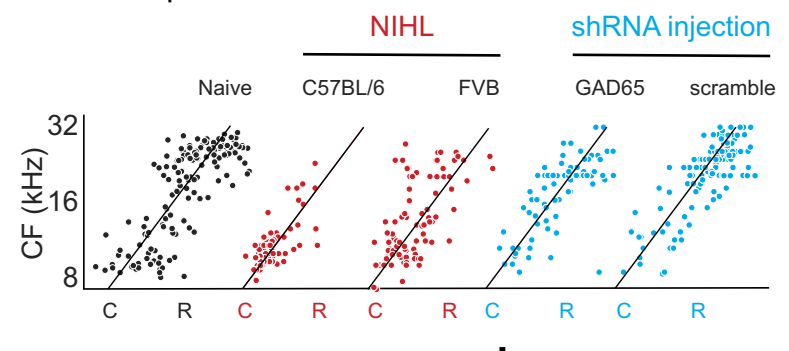

C $8-16 \mathrm{kHz}$ representation

d Al Threshold

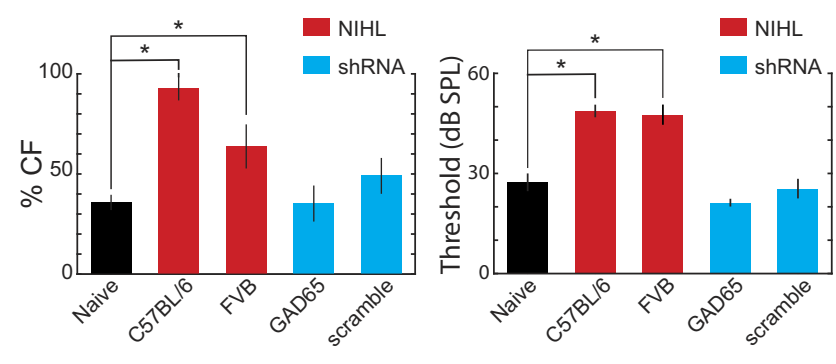

Figure 5. NIHL causes Al reorganization and increases firing threshold. $\boldsymbol{a}$, Top, Example Al

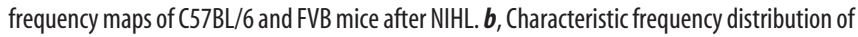
all subjects along the caudal-rostral $(C-R)$ extent of Al. Each dot indicates a multiunit. c, Percentage of $8-16 \mathrm{kHz}$ tuned sites in. $\boldsymbol{d}$, Al multiunit response threshold. Error bars represent \pm SEM. ${ }^{*} p<0.05$.

\section{Tinnitus is not associated with distortion in the AI frequency map}

Our findings raise the question of how GAD65 downregulation affects the primary AI sensory map and the response properties of AI neurons. To address this, we conducted AI recordings in noise-exposed and virus-injected FVB and C57BL/6 mice using pure tones spanning 4 to $64 \mathrm{kHz}$.

The tonotopy of naive mice consists of an orderly caudalrostral representation spanning $8-32 \mathrm{kHz}$ (Fig. 5a,b). NIHL expanded the AI frequency representation in the octave $>8 \mathrm{kHz}$, the center frequency of the noise. Frequency representation was characterized by the proportion of multiunits tuned to a low $(8-16 \mathrm{kHz})$ and high $(16-32 \mathrm{kHz})$ octave range. In C57BL/6 and FVB mice with NIHL, the NIHL-affected (right) AI of both strains showed increased low-frequency $(8-16 \mathrm{kHz})$ representation relative to the naive AI (Fig. $5 b, c$, naive vs C57BL/6 NIHL $t_{7}$ $=8.136, p<0.001$, naive vs FVB NIHL $\left.t_{6}=2.46, p=0.047\right)$. We did not observe a broadening of frequency tuning toward low frequencies. Instead, this frequency map change was a result of increased low-frequency responses and decreased high-frequency responses. In contrast to the altered frequency map organization following NIHL, change in the AI map was absent in virusinjected FVB mice regardless of the virus type (GAD65 shRNA or scrambled shRNA). Orderly tonotopy spanning $8-32 \mathrm{kHz}$ was maintained in both groups.

Multiunit response thresholds for AI of the right hemisphere were elevated by NIHL in both C57BL/6 and FVB mice (Fig. $5 d$ naive vs C57BL6 NIHL $t_{7}=7.65, p<0.001$, naive vs FVB NIHL $\left.t_{6}=5.55, p=0.001\right)$. Threshold change was absent in FVB mice that underwent cortical virus injection (Fig. $5 d$ ).

In summary, NIHL distorted the AI frequency map and raised multiunit response thresholds. While these changes are present in both strains, only C57BL/6 developed tinnitus. This result suggests that NIHL, but not tinnitus, is associated with AI changes. In addition, FVB mice with cortical GAD65 knock-down developed tinnitus despite the absence of the map change. This dissociation further confirms that sensory map reorganizations are not necessary for tinnitus development.

\section{GAD65 knock-down reduces the AI receptive field size}

Acute pharmacological blockage of GABA synapses has been shown to increase responsivity in both spectral and intensity dimensions (Wang et al., 2002). However, cortex-specific chronic reduction of inhibition has been shown to reduce the receptive field size (Seybold et al., 2012). To examine the spectral-intensity responsiveness following a localized downregulation by shRNA, we quantified the median frequency response area (FRA) size. The AI with shRNA-mediated GAD65 reduction showed smaller threshold-adjusted FRAs than both the AI of the uninjected side and AI of naive animals $\left(t_{5}=3.21, p=0.02, t_{8}=\right.$ 2.37, $p=0.045$, respectively; Fig. $6 a$ ). Similar reductions of FRA have been reported in mice with chronic reduction of cortical inhibition (Seybold et al., 2012).

Evoked firing was defined as the multiunits' mean firing in the duration where the PSTH is above half of the maximum. Both evoked and spontaneous firing rate were similar to naive cortex. However, differences were observed in bilateral comparisons (Left vs Right cortex of the same animals) for some conditions. In noise-exposed C57BL/6 and FVB mice, the NIHL-affected (right) AI showed significantly lower evoked FR compared with the left AI (ANOVA, evoked FR: $F_{(1,7)}=36.40, p=0.001$, post hoc paired $t$ test, C57BL/6: $t_{4}=4.01, p=0.02$, FVB: $t_{3}=8.29, p<0.01$; Fig. $6 b)$. The reduction of evoked FR was equivalent between strains (ANOVA, evoked FR-by-strain interaction: $F_{(1,7)}=0.83, p=$ $0.39)$. In GAD65 knock-down and scrambled-sequence control mice, no statistically significant difference in evoked firing rates was found between the injected and uninjected sides (ANOVA, evoked FR: $F_{(1,9)}=0.76, p=0.4$; Fig. $\left.6 b\right)$.

Spontaneous firing was recorded in a silent 5 min block following the sound stimulation block, while maintaining the same electrode locations and the spike threshold. AI multiunits with CFs between 8 and $32 \mathrm{kHz}$ were included in the calculation of spontaneous rate. A reduction in spontaneous firing rate was observed in the cortical side with GAD65 downregulation, compared with the intact side- both in the NIHL case and artificial GAD65 knock-down case (C57BL/6: $t_{4}=2.94, p=0.04$, FVB GAD65: $t_{5}=3.40, p=0.02$, paired $t$ test; Fig. $6 c$ ). A similar reduction in spontaneous FR was confirmed in the sound presentation blocks when FR was evaluated in the $50 \mathrm{~ms}$ silent period immediately preceding the stimulus presentation $\left(\mathrm{C} 57 \mathrm{BL} / 6: t_{4}=\right.$ 2.61, $p=0.03$, FVB GAD65: $t_{5}=2.76, p=0.02$, one-tailed paired $t$ test). Thus, reduction in spontaneous firing was observed both 
in prolonged $(5 \mathrm{~min})$ and short $(<333$ $\mathrm{ms}$ ) silent periods. Results of this study are summarized in Table 1.

\section{Discussion}

Neuronal inhibition is reduced in many auditory brain regions in animals with hearing loss-induced tinnitus (Abbott et al., 1999; Milbrandt et al., 2000; Kotak et al., 2005; Dong et al., 2009, 2010; Roberts et al., 2010; Kaltenbach, 2011; Middleton et al., 2011; Wang et al., 2011; Browne et al., 2012; Llano et al., 2012; Takesian et al., 2013). In this study, we found that: (1) cortical downregulation of GAD65 expression was correlated with the severity of tinnitus as measured by two behavioral tasks; (2) localized knock-down of cortical GAD65, to a level that was observed in noise-exposed mice, caused tinnitus in normal-hearing animals; and (3) animals showing cortical map reorganization did not necessarily exhibit tinnitus, and animals exhibiting tinnitus did not consistently show map reorganization. These results suggest a critical role of cortical GAD65 downregulation in noise-induced tinnitus (Yang et al., 2011; Llano et al., 2012).

Sensory map reorganization has been considered as a potential mechanism underlying tinnitus, since abnormal AI activation and cortical map reorganization are correlated with the occurrence and severity of tinnitus in human patients and in animal models (Mühlnickel et al., 1998; Noreña and Eggermont, 2005). In addition, hearing loss associated with tinnitus leads to altered spontaneous activity and map reorganization, both of which can be prevented if the trauma is followed by enriched acoustic experience (Komiya and Eggermont, 2000; Seki and Eggermont, 2003; Noreña and Eggermont, 2005; Engineer et al., 2011). In our study, both C57BL/6 and FVB mice that underwent noise exposure showed an expansion of low $(8-16 \mathrm{kHz})$ frequency representation but only C57BL/6 mice developed tinnitus. Further, GAD65 knock-down mice that also had tinnitus did not show sensory map changes. Therefore, sensory map change is likely causally related to the hearing loss, but not to tinnitus. Our results are consistent with recent findings that macroscopic sensory map changes are not correlated with tinnitus (Langers et al., 2012; Ghazaleh et al., 2017).

The GAD 65 protein accounts for $\sim 50 \%$ of the total amount of GAD expressed in the cerebral cortex (Sheikh et al., 1999). GAD65 expression is preferentially localized in axonal terminals, is modulated by activity and sensory input, and influences the amount of GABA release and the level of neuronal activity (Esclapez et al., 1994; Patel et al., 2006). Complete knock-out of the GAD65 gene leads to epileptic activity in the neocortex, indicative of a general increase in neuronal excitability (Kash et al., 1997). Here we show that a 40\% GAD65 reduction by virus-mediated gene knock-down in AI is sufficient to cause tinnitus.

A potential consequence of such a drastic reduction of GAD65 expression in the cortex is reduced GABA release and associated

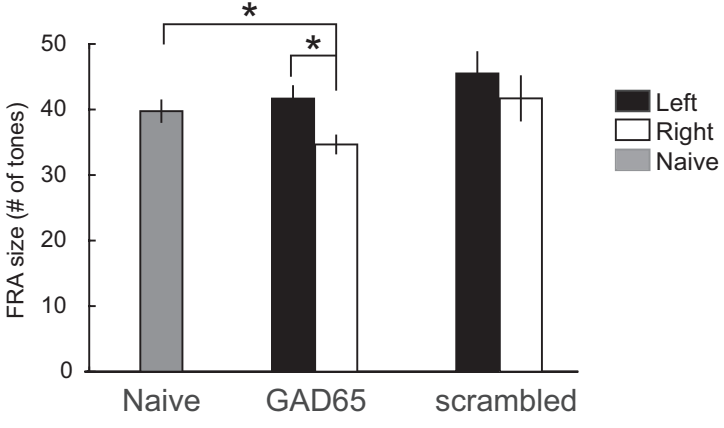

c Spontaneous FR

\section{b Evoked FR}

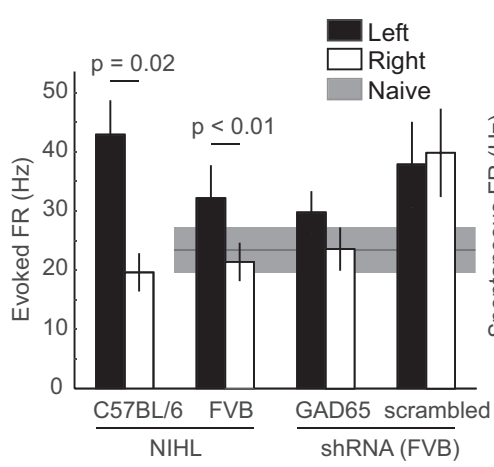

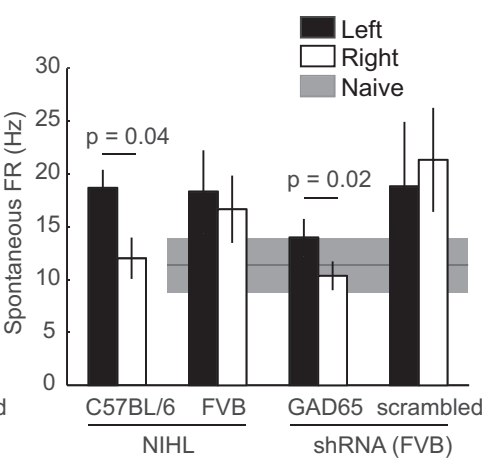

Figure 6. GAD65 knock-down decreases receptive field size. Quantification of $\boldsymbol{a}$, Al FRA size. Shown is quantification of evoked firing rate $(\boldsymbol{b})$ and spontaneous firing rate $(\boldsymbol{c})$ in four groups and two cortical sides. Right cortex was the target of manipulation in both NIHL and shRNA experiments. Left cortex was the same animal. Black line and surrounding gray shading in the background indicates mean \pm SEM of the firing rate for evoked $(\boldsymbol{a})$ or spontaneous $(\boldsymbol{b})$ conditions in naive FVB mice $(n=6$, reanalysis of a previously published dataset; Kim et al., 2013). Error bars represent \pm SEM. ${ }^{*} p<0.05$.

reductions in phasic and tonic inhibition (Yang et al., 2011). Reduced tonic inhibition may lead to higher input resistance and increased neuronal excitability (Yang et al., 2012). However, in this study, animals with GAD65 knock-down showed lower spontaneous activity and smaller receptive field size in the AI $20-30 \mathrm{~d}$ after the manipulation. Although unexpected, it is possible that GAD65 downregulation is followed by secondary postsynaptic compensatory changes, such as sensitization of GABA receptors through modification of subunit compositions and functions as previously reported (Caspary et al., 1999). In addition, our findings are consistent with those of a recent report showing reduced FRA size after chronic reduction in cortical inhibition in conditional Dlx knock-out mice (Seybold et al., 2012). Together, these results suggest that acute and chronic reduction in inhibition may differentially affect cortical excitability and responses. Cellular mechanisms underlining our spontaneous firing rate results remain to be determined. In the present study, the effects of NIHL and GAD65 knock-down were examined 10-14 d after the manipulations. Tinnitus assessed 2 weeks after noise exposure was considered chronic (Zheng et al., 2012). This time span also allows homeostatic adjustment of neuronal circuits (Keck et al., 2013; Wang et al., 2019), which are considered a potential mechanism for tinnitus (Yang et al., 2011). Additional effects of these manipulations on a longer term remain to be determined.

In our study, whereas reduced sound-evoked responses were correlated with hearing loss, reduced spontaneous activity appeared to be correlated with tinnitus. The results were surprising given that tinnitus had previously been associated with increased 
Table 1. Summary of results

\begin{tabular}{|c|c|c|c|c|c|c|c|}
\hline Strain & Treatment & Tinnitus & Hearing loss & Map change & Evoked FR & Spontaneous FR & GAD65 \\
\hline (57BL/6] & NIHL & + & + & + & $+\downarrow$ & $+\downarrow$ & $+\downarrow$ \\
\hline FVB0.129P2-Pde6b + Tyrc-ch/AntJ & NIHL & - & + & + & $+\downarrow$ & - & - \\
\hline FVB0.129P2-Pde6b + Tyrc-ch/AntJ & GAD65 ShRNA & + & - & - & - & $+\downarrow$ & $+\downarrow$ \\
\hline FVB0.129P2-Pde6b + Tyrc-ch/AntJ & scrambled shRNA & - & - & - & - & - & - \\
\hline $\operatorname{Tg}(\mathrm{dl} x 6 \mathrm{a}-\mathrm{cre}) 1$ Mekk & NIHL & + & + & N/A & $\mathrm{N} / \mathrm{A}$ & $\mathrm{N} / \mathrm{A}$ & $+\downarrow$ \\
\hline Tg(dlx6a-cre)1Mekk & GAD65 ShRNA & + & - & $\mathrm{N} / \mathrm{A}$ & $\mathrm{N} / \mathrm{A}$ & N/A & $+\downarrow$ \\
\hline
\end{tabular}

Positive sign (+) indicates the presence of a statistically significant difference compared with the appropriate control condition. Negative sign (-) indicates the absence of a statistically significant difference compared with the contro condition. When applicable, the direction of the positive result is indicated with an arrow. Down arrow $(\downarrow)$ indicates a reduction. N/A, Data not available.

spontaneous activity (Komiya and Eggermont, 2000; Seki and Eggermont, 2003; Schaette and Kempter, 2006; Roberts et al., 2010; Kaltenbach, 2011). The fact that reduced spontaneous activity occurred in both noise-exposed C57BL/6 and GAD65knock-down FVB mice suggests that the result was not due to a specific type of manipulation. We should be cautious interpreting these results because the recordings were acquired mostly from Layer 4 under anesthesia and may not directly relate to tinnitus behavior. However, several previous studies have also reported reduced spontaneous cortical activity and local field potential power in awake animals after tinnitus induction (Yang et al., 2007; Noreña et al., 2010).

Human genetic studies have indicated low heritability of tinnitus (Kvestad et al., 2010), which is consistent with a major role played by environmental factors - such as noise trauma and ototoxic drug use-in tinnitus etiology (Henry et al., 2005). However, since the causes of tinnitus are heterogeneous, the low overall heritability does not exclude the possibility of higher heritability of some subtypes of tinnitus. Furthermore, low heritability of tinnitus does not exclude the possibility of higher heritability of resistance to hearing-loss-related tinnitus, as we have seen in the FVB mice. Tinnitus is linked to several genes (Sand et al., 2007, 2012a,b), some of which, such as the auxiliary GABA-B receptor subunit gene potassium channel tetramerization domain containing 12 (KCTD12) and the brain-derived neurotrophic factor (BDNF) gene, are linked to the function of inhibitory neurons (Huang et al., 1999; Sand et al., 2012a,b). It remains to be determined whether the GAD65 gene and its regulation mechanisms are linked to tinnitus or its resistance in humans.

In contrast to the large strain differences in hearing lossinduced tinnitus, within-strain variability in our study is small. All the eight C57BL/ 6 with NIHL showed impaired gap detection and a sign of tinnitus. Previous studies reported higher intersubject variability. For example, not all animals with NIHL exhibit behavioral signs of tinnitus (Middleton et al., 2011). Although the precise causes of this discrepancy are unknown, potential source of variability may be the degree of hearing impairment introduced to the animals. To avoid the confounding factor of hearing loss in the measurement of tinnitus, researchers often choose mild, transient or spectrally narrow-range hearing loss in model animals. Animals in those studies may be close to a threshold of tinnitus development, resulting in only a fraction showing measurable signs of tinnitus. This notion is supported by findings that tinnitus behaviors are correlated with the degree of persistent hearing loss in animal models (Tan et al., 2007). The hearing lesion procedure of prolonged noise exposure in our study may have resulted in more consistent hearing impairment and thus the induction of tinnitus.

Impairment in gap-induced prepulse inhibition has been correlated with behavioral evidence of tinnitus assessed with other behavioral tests in animal models (Turner et al., 2006; Yang et al.,
2007; Pace and Zhang, 2013), and consistent patterns of impairment in the task have been shown in human tinnitus patients (Fournier and Hébert, 2013). The initial notion that tinnitus "fills the gap" has been challenged by findings that tinnitus patients can perceive the gap (Mehdizade Gilani et al., 2013; Boyen et al., 2015; Galazyuk and Hébert, 2015). It is possible that tinnitus and impairment in gap-induced prepulse inhibition share similar pathological mechanisms, such as reduced cortical inhibitory neuronal functions (Yang et al., 2011; Weible et al., 2014; Keller et al., 2018). Another major concern about the task is whether the gap detection behavior is altered by hearing loss rather than, or in addition to, tinnitus (Lobarinas et al., 2013). In our study, we observed no impairment in gap detection in FVB mice that had substantial monaural hearing loss. In contrast, the FVB mice that had cortical GAD65 knock-down were impaired in gap detection without changes in the ABR thresholds, peak latency, and peak magnitude. The double-dissociation between hearing loss and impaired gap detection indicate that monaural hearing loss does not necessarily impair gap detection. A third question about gap detection is whether it is modulated by thalamocortical circuits (Kaltenbach, 2011; Eggermont, 2013). Previous cortical deactivation studies have suggested cortical involvement in resolving short duration $(<75 \mathrm{~ms}$ ) silent gap detection in rodents (Syka et al., 2002; Threlkeld et al., 2008). Recent studies implicated cortical PV-positive inhibitory interneurons in representing gaps in sound (Weible et al., 2014; Keller et al., 2018). In our study, FVB mice with cortical knock-down of GAD65 are significantly impaired in gap detection. These results suggest that gap detection is modulated by the AI. A fourth concern with the gap detection is the reduction of acoustic startle responses after NIHL (Lobarinas et al., 2013). We have observed similar reduction of startle responses after NIHL. However, the startle response amplitude did not correlate with the tinnitus score, thus the reduction in startle amplitude was unlikely to have confounded our tinnitus measurements.

Despite intense research efforts, the neural substrates and mechanisms underlying hearing loss-induced tinnitus have so far been elusive. A major obstacle has been the difficulty in dissociating the mechanisms underlying tinnitus from consequences of hearing loss. Taking advantage of the strain differences between $\mathrm{C} 57 \mathrm{BL} / 6$ and FVB mice, we provide evidences that the $\mathrm{AI}$ is part of the circuit involved in hearing loss-induced tinnitus, and that homeostatic reduction of cortical GAD65 expression plays a pivotal role in tinnitus etiology (Noreña and Eggermont, 2005; Eggermont, 2008; Engineer et al., 2011; Yang et al., 2011; Llano et al., 2012; Yang and Bao, 2013). In addition, sensory map reorganization is likely a consequence of altered sensory inputs rather than a cause of the phantom sensation, as has been widely hypothesized (Flor et al., 1995). These findings suggest that new strategies should be sought for rehabilitation of phantom sensations. 


\section{References}

Abbott SD, Hughes LF, Bauer CA, Salvi R, Caspary DM (1999) Detection of glutamate decarboxylase isoforms in rat inferior colliculus following acoustic exposure. Neuroscience 93:1375-1381.

Bauer CA, Brozoski TJ (2001) Assessing tinnitus and prospective tinnitus therapeutics using a psychophysical animal model. J Assoc Res Otolaryngol 2:54-64.

Boyen K, Başkent D, van Dijk P (2015) The gap detection test: can it be used to diagnose tinnitus? Ear Hear 36:e138-e145.

Browne CJ, Morley JW, Parsons CH (2012) Tracking the expression of excitatory and inhibitory neurotransmission-related proteins and neuroplasticity markers after noise induced hearing loss. PLoS One 7:e33272.

Caspary DM, Holder TM, Hughes LF, Milbrandt JC, McKernan RM, Naritoku DK (1999) Age-related changes in GABA(A) receptor subunit composition and function in rat auditory system. Neuroscience 93:307-312.

Chambers AR, Resnik J, Yuan Y, Whitton JP, Edge AS, Liberman MC, Polley DB (2016) Central gain restores auditory processing following nearcomplete cochlear denervation. Neuron 89:867-879.

Davis RR, Cheever ML, Krieg EF, Erway LC (1999) Quantitative measure of genetic differences in susceptibility to noise-induced hearing loss in two strains of mice. Hear Res 134:9-15.

Dong S, Mulders WH, Rodger J, Robertson D (2009) Changes in neuronal activity and gene expression in guinea-pig auditory brainstem after unilateral partial hearing loss. Neuroscience 159:1164-1174.

Dong S, Rodger J, Mulders WH, Robertson D (2010) Tonotopic changes in GABA receptor expression in guinea pig inferior colliculus after partial unilateral hearing loss. Brain Res 1342:24-32.

Eggermont JJ (2008) Role of auditory cortex in noise- and drug-induced tinnitus. Am J Audiol 17:S162-S169.

Eggermont JJ (2013) Hearing loss, hyperacusis, or tinnitus: What is modeled in animal research? Hear Res 295:140-149.

Eggermont JJ, Roberts LE (2004) The neuroscience of tinnitus. Trends Neurosci 27:676-682.

Elgoyhen AB, Langguth B (2010) Pharmacological approaches to the treatment of tinnitus. Drug Discov Today 15:300-305.

Engineer ND, Riley JR, Seale JD, Vrana WA, Shetake JA, Sudanagunta SP, Borland MS, Kilgard MP (2011) Reversing pathological neural activity using targeted plasticity. Nature 470:101-104.

Esclapez M, Tillakaratne NJ, Kaufman DL, Tobin AJ, Houser CR (1994) Comparative localization of two forms of glutamic acid decarboxylase and their mRNAs in rat brain supports the concept of functional differences between the forms. J Neurosci 14:1834-1855.

Flor H, Elbert T, Knecht S, Wienbruch C, Pantev C, Birbaumer N, Larbig W, Taub E (1995) Phantom-limb pain as a perceptual correlate of cortical reorganization following arm amputation. Nature 375:482-484.

Fournier P, Hébert S (2013) Gap detection deficits in humans with tinnitus as assessed with the acoustic startle paradigm: does tinnitus fill in the gap? Hear Res 295:16-23.

Galazyuk A, Hébert S (2015) Gap-prepulse inhibition of the acoustic startle reflex (GPIAS) for tinnitus assessment: current status and future directions. Front Neurol 6:88.

Ghazaleh N, Zwaag WV, Clarke S, Ville DV, Maire R, Saenz M (2017) Highresolution fMRI of auditory cortical map changes in unilateral hearing loss and tinnitus. Brain Topogr 30:685-697.

Heffner HE, Koay G (2005) Tinnitus and hearing loss in hamsters (Mesocricetus auratus) exposed to loud sound. Behav Neurosci 119:734-742.

Henry JA, Dennis KC, Schechter MA (2005) General review of tinnitus: prevalence, mechanisms, effects, and management. J Speech Lang Hear Res 48:1204-1235.

Huang ZJ, Kirkwood A, Pizzorusso T, Porciatti V, Morales B, Bear MF, Maffei L, Tonegawa S (1999) BDNF regulates the maturation of inhibition and the critical period of plasticity in mouse visual cortex. Cell 98:739-755.

Kaltenbach JA (2011) Tinnitus: models and mechanisms. Hear Res 276:5260.

Kash SF, Johnson RS, Tecott LH, Noebels JL, Mayfield RD, Hanahan D, Baekkeskov S (1997) Epilepsy in mice deficient in the 65-kDa isoform of glutamic acid decarboxylase. Proc Natl Acad Sci U S A 94:14060-14065.

Keck T, Keller GB, Jacobsen RI, Eysel UT, Bonhoeffer T, Hübener M (2013) Synaptic scaling and homeostatic plasticity in the mouse visual cortex in vivo. Neuron 80:327-334.
Keller CH, Kaylegian K, Wehr M (2018) Gap encoding by parvalbuminexpressing interneurons in auditory cortex. J Neurophysiol 120:105-114.

Kim H, Gibboni R, Kirkhart C, Bao S (2013) Impaired critical period plasticity in primary auditory cortex of fragile $\mathrm{x}$ model mice. J Neurosci 33:15686-15692.

Komiya H, Eggermont JJ (2000) Spontaneous firing activity of cortical neurons in adult cats with reorganized tonotopic map following pure-tone trauma. Acta Otolaryngol 120:750-756.

Kotak VC, Fujisawa S, Lee FA, Karthikeyan O, Aoki C, Sanes DH (2005) Hearing loss raises excitability in the auditory cortex. J Neurosci 25:3908-3918.

Kujawa SG, Liberman MC (2009) Adding insult to injury: cochlear nerve degeneration after "temporary" noise-induced hearing loss. J Neurosci 29:14077-14085.

Kvestad E, Czajkowski N, Engdahl B, Hoffman HJ, Tambs K (2010) Low heritability of tinnitus: results from the second nord-trondelag health study. Arch Otolaryngol Head Neck Surg 136:178-182.

Langers DR, de Kleine E, van Dijk P (2012) Tinnitus does not require macroscopic tonotopic map reorganization. Front Syst Neurosci 6:2.

Langguth B, Kreuzer PM, Kleinjung T, De Ridder D (2013) Tinnitus: causes and clinical management. Lancet Neurol 12:920-930.

Liberman MC, Kiang NY (1978) Acoustic trauma in cats. cochlear pathology and auditory-nerve activity. Acta Otolaryngol Suppl 358:1-63.

Llano DA, Turner J, Caspary DM (2012) Diminished cortical inhibition in an aging mouse model of chronic tinnitus. J Neurosci 32:16141-16148.

Lobarinas E, Sun W, Cushing R, Salvi R (2004) A novel behavioral paradigm for assessing tinnitus using schedule-induced polydipsia avoidance conditioning (SIP-AC). Hear Res 190:109-114.

Lobarinas E, Hayes SH, Allman BL (2013) The gap-startle paradigm for tinnitus screening in animal models: Limitations and optimization. Hear Res 295:150-160.

Lockwood AH, Salvi RJ, Burkard RF (2002) Tinnitus. N Engl J Med 347: 904-910.

Longenecker RJ, Galazyuk AV (2011) Development of tinnitus in CBA/CaJ mice following sound exposure. J Assoc Res Otolaryngol 12:647-658.

Mehdizade Gilani V, Ruzbahani M, Mahdi P, Amali A, Nilforush Khoshk MH, Sameni J, Karimi Yazdi A, Emami H (2013) Temporal processing evaluation in tinnitus patients: results on analysis of gap in noise and duration pattern test. Iran J Otorhinolaryngol 25:221-226.

Middleton JW, Kiritani T, Pedersen C, Turner JG, Shepherd GM, Tzounopoulos T (2011) Mice with behavioral evidence of tinnitus exhibit dorsal cochlear nucleus hyperactivity because of decreased GABAergic inhibition. Proc Natl Acad Sci U S A 108:7601-7606.

Milbrandt JC, Holder TM, Wilson MC, Salvi RJ, Caspary DM (2000) GAD levels and muscimol binding in rat inferior colliculus following acoustic trauma. Hear Res 147:251-260.

Møller AR (2011) Epidemiology of tinnitus in adults. In: Textbook of tinnitus (Møller AR, Langguth B, Ridder D, Kleinjung T, eds), pp 29-37. New York: Springer.

Mühlnickel W, Elbert T, Taub E, Flor H (1998) Reorganization of auditory cortex in tinnitus. Proc Natl Acad Sci U S A 95:10340-10343.

Noreña AJ, Eggermont JJ (2003) Changes in spontaneous neural activity immediately after an acoustic trauma: implications for neural correlates of tinnitus. Hear Res 183:137-153.

Noreña AJ, Eggermont JJ (2005) Enriched acoustic environment after noise trauma reduces hearing loss and prevents cortical map reorganization. J Neurosci 25:699-705.

Noreña AJ, Moffat G, Blanc JL, Pezard L, Cazals Y (2010) Neural changes in the auditory cortex of awake guinea pigs after two tinnitus inducers: salicylate and acoustic trauma. Neuroscience 166:1194-1209.

Pace E, Zhang J (2013) Noise-induced tinnitus using individualized gap detection analysis and its relationship with hyperacusis, anxiety, and spatial cognition. PLoS One 8:e75011.

Patel AB, de Graaf RA, Martin DL, Battaglioli G, Behar KL (2006) Evidence that GAD65 mediates increased GABA synthesis during intense neuronal activity in vivo. J Neurochem 97:385-396.

Ranson A, Cheetham CE, Fox K, Sengpiel F (2012) Homeostatic plasticity mechanisms are required for juvenile, but not adult, ocular dominance plasticity. Proc Natl Acad Sci U S A 109:1311-1316.

Resnik J, Polley DB (2017) Fast-spiking GABA circuit dynamics in the auditory cortex predict recovery of sensory processing following peripheral nerve damage. Elife 6:e21452. 
Roberts LE, Eggermont JJ, Caspary DM, Shore SE, Melcher JR, Kaltenbach JA (2010) Ringing ears: the neuroscience of tinnitus. J Neurosci 30:14972-14979.

Salvi RJ, Wang J, Ding D (2000) Auditory plasticity and hyperactivity following cochlear damage. Hear Res 147:261-274.

Sand PG, Langguth B, Kleinjung T, Eichhammer P (2007) Genetics of chronic tinnitus. Prog Brain Res 166:159-168.

Sand PG, Langguth B, Schecklmann M, Kleinjung T (2012a) GDNF and BDNF gene interplay in chronic tinnitus. Int J Mol Epidemiol Genet 3:245-251.

Sand PG, Langguth B, Itzhacki J, Bauer A, Geis S, Cárdenas-Conejo ZE, Pimentel V, Kleinjung T (2012b) Resequencing of the auxiliary GABA(B) receptor subunit gene KCTD12 in chronic tinnitus. Front Syst Neurosci 6:41.

Schaette R, Kempter R (2006) Development of tinnitus-related neuronal hyperactivity through homeostatic plasticity after hearing loss: a computational model. Eur J Neurosci 23:3124-3138.

Seki S, Eggermont JJ (2003) Changes in spontaneous firing rate and neural synchrony in cat primary auditory cortex after localized tone-induced hearing loss. Hear Res 180:28-38.

Seybold BA, Stanco A, Cho KK, Potter GB, Kim C, Sohal VS, Rubenstein JL, Schreiner CE (2012) Chronic reduction in inhibition reduces receptive field size in mouse auditory cortex. Proc Natl Acad Sci USA 109:13829-13834.

Sheikh SN, Martin SB, Martin DL (1999) Regional distribution and relative amounts of glutamate decarboxylase isoforms in rat and mouse brain. Neurochem Int 35:73-80.

Shore SE, Roberts LE, Langguth B (2016) Maladaptive plasticity in tinnitustriggers, mechanisms and treatment. Nat Rev Neurol 12:150-160.

Sun W, Zhang L, Lu J, Yang G, Laundrie E, Salvi R (2008) Noise exposureinduced enhancement of auditory cortex response and changes in gene expression. Neuroscience 156:374-380.

Syka J, Rybalko N, Mazelová J, Druga R (2002) Gap detection threshold in the rat before and after auditory cortex ablation. Hear Res 172:151-159.

Takesian AE, Kotak VC, Sharma N, Sanes DH (2013) Hearing loss differentially affects thalamic drive to two cortical interneuron subtypes. J Neurophysiol 110:999-1008.

Tan J, Rüttiger L, Panford-Walsh R, Singer W, Schulze H, Kilian SB, Hadjab S, Zimmermann U, Köpschall I, Rohbock K, Knipper M (2007) Tinnitus behavior and hearing function correlate with the reciprocal expression patterns of BDNF and Arg3.1/arc in auditory neurons following acoustic trauma. Neuroscience 145:715-726.

Threlkeld SW, Penley SC, Rosen GD, Fitch RH (2008) Detection of silent gaps in white noise following cortical deactivation in rats. Neuroreport 19:893-898.

Turner JG, Brozoski TJ, Bauer CA, Parrish JL, Myers K, Hughes LF, Caspary DM (2006) Gap detection deficits in rats with tinnitus: a potential novel screening tool. Behav Neurosci 120:188-195.

Wang H, Brozoski TJ, Caspary DM (2011) Inhibitory neurotransmission in animal models of tinnitus: maladaptive plasticity. Hear Res 279:111-117.

Wang J, McFadden SL, Caspary D, Salvi R (2002) Gamma-aminobutyric acid circuits shape response properties of auditory cortex neurons. Brain Res 944:219-231.

Wang W, Zhang LS, Zinsmaier AK, Patterson G, Leptich EJ, Shoemaker SL, Yatskievych TA, Gibboni R, Pace E, Luo H, Zhang J, Yang S, Bao S (2019) Neuroinflammation mediates noise-induced synaptic imbalance and tinnitus in rodent models. PLoS Biol 17:e3000307.

Weible AP, Moore AK, Liu C, DeBlander L, Wu H, Kentros C, Wehr M (2014) Perceptual gap detection is mediated by gap termination responses in auditory cortex. Curr Biol 24:1447-1455.

Wu C, Martel DT, Shore SE (2016) Increased synchrony and bursting of dorsal cochlear nucleus fusiform cells correlate with tinnitus. J Neurosci 36:2068-2073.

Yang G, Lobarinas E, Zhang L, Turner J, Stolzberg D, Salvi R, Sun W (2007) Salicylate induced tinnitus: behavioral measures and neural activity in auditory cortex of awake rats. Hear Res 226:244-253.

Yang S, Bao S (2013) Homeostatic mechanisms and treatment of tinnitus. Restor Neurol Neurosci 31:99-108.

Yang S, Weiner BD, Zhang LS, Cho SJ, Bao S (2011) Homeostatic plasticity drives tinnitus perception in an animal model. Proc Natl Acad Sci U S A 108:14974-14979.

Yang S, Su W, Bao S (2012) Long-term, but not transient, threshold shifts alter the morphology and increase the excitability of cortical pyramidal neurons. J Neurophysiol 108:1567-1574.

Zheng QY, Johnson KR, Erway LC (1999) Assessment of hearing in 80 inbred strains of mice by ABR threshold analyses. Hear Res 130:94-107.

Zheng Y, Vagal S, McNamara E, Darlington CL, Smith PF (2012) A doseresponse analysis of the effects of L-baclofen on chronic tinnitus caused by acoustic trauma in rats. Neuropharmacology 62:940-946. 